\title{
A DECOUPLING FINITE ELEMENT METHOD WITH DIFFERENT TIME STEPS FOR THE MICROPOLAR FLUID MODEL*
}

\author{
PENGZHAN HUANG ${ }^{\dagger}$ AND CHENG LIAO ${ }^{\ddagger}$
}

\begin{abstract}
In this paper, a decoupling finite element method with different time steps for the micropolar fluid model is considered. The theoretical analysis shows that the proposed method is stable and convergent. Further, in order to show the efficiency of the method, we present some numerical results for a problem with analytical solution, and we test the method for the stirring problem of a passive scalar. From these numerical results, we can see that the method is efficient for micropolar fluid flows.
\end{abstract}

Key words. finite element method, decoupling method, different time steps, micropolar fluid model

AMS subject classifications. 65M12, 65M15, 65M60

1. Introduction. The micropolar fluid model, introduced by Eringen [10, 11], can simulate the interaction between the evolution of an incompressible fluid and the rotational motion of material microparticles that are suspended in a viscous medium and possess rotational degrees of freedom. Hence, this is a very attractive model for the dynamical description of continuum media subject to distributed couples and for polar media in general.

As is known, one of the fundamental assumptions in fluid mechanics is that material particles do not possess an angular momentum (the stress tensor is symmetric). However, this assumption does not hold when microscopic gyration effects become important (the stress tensor is then nonsymmetric). For example, the angular momentum should be taken explicitly into account to describe the behavior of micro- and nanoflows, to effectively design and fabricate microchannels and chambers for microfluidic systems [29, 40], and to represent anisotropic fluids (e.g., animal blood, exotic lubricants, and liquid crystals which are made up of dumbbell molecules) [11, 24]. In fact, in the micropolar fluid model, the incompressible Navier-Stokes equations is coupled with micro-rotational effects and micro-rotational inertia. Hence, this model is more suitable in describing and representing the behavior of flows in microfluidic systems than the classical Navier-Stokes equations, and it has a great deal of practical applications in many current scientific, engineering, and industrial problems [1,24].

Because of the mathematical and engineering importance of the micropolar fluid model, there are numerous papers devoted to the model's mathematical analysis, such as existence, uniqueness, and regularity of solutions to micropolar flow equations; see [18, 24, 32, 34, 43]. Łukaszewicz [23] has established the local existence of weak solutions by using a linearization and an almost fixed-point theorem. In [5], using a spectral semi-Galerkin method, the existence of a local-in-time strong solution, the uniqueness of the strong solution, and some global existence results have been proved. Galdi and Rionero [12] have shown that the weak form of the unsteady incompressible micropolar fluid equations belongs to a general class of evolution problems which the Navier-Stokes equations belong to as well, and the authors studied existence and uniqueness results for the weak solutions of the considered problem. Stability problems for micropolar fluids have been investigated in [30]. For the long time behavior of the two-dimensional micropolar fluid system, the existence of $L^{2}$-global attractors in a bounded domain has been obtained [25]. Chen and Price [6] have shown the existence and

* Received January 11, 2021. Accepted December 6, 2021. Published online on January 14, 2022. Recommended by Bill Layton.

$\dagger$ College of Mathematics and System Sciences, Xinjiang University, Urumqi 830046, P.R. China (hpzh007@yahoo.com).

${ }^{\ddagger}$ Department of Mathematics, Taiyuan University of Technology, Taiyuan 030024, P.R. China (liaoc123@163.com). 
uniqueness of small $L^{3}$-strong solutions of the micropolar fluid motion system and derived some sharp time decay estimates of strong $L^{3}$-solutions.

Since the governing equations of the micropolar fluid model include not only incompressibility and a strong nonlinearity but also the coupling between the angular motion equation and the fluid equations of motion, it is not easy to solve these equations effectively. Therefore, much effort has been invested into the development of efficient numerical methods for investigating this problem. For the spectral Galerkin approach of a spatial semi-discretization of the micropolar fluid model, Boldrini and Rojas-Medar [4] have studied the convergence rate of approximate solutions. Further, they have obtained a pointwise convergence rate without any compatibility conditions for the initial data [3]. A decoupled time-stepping finite element scheme for the evolutionary micropolar fluid flow model has been proposed and studied in [31], where the Navier-Stokes equations and the microrotational velocity equations are solved separately in each time step without iteration. Nochetto et al. [26] have developed a semi-implicit fully discrete scheme which, at each time step, decouples the computation of the linear and angular velocities but requires the solution of a saddle-point problem for the determination of the linear velocity and the pressure. In order to remedy this shortcoming, by applying a fractional time stepping technique, Salgado [33] has developed an unconditionally stable first-order scheme and an almost unconditionally stable second-order scheme. In [27], a fully discrete penalty finite element method has been proposed and analyzed, and suboptimal error estimates have been proved. Yang and Jiang [42] have proposed and analyzed some projection methods including first-order and second-order projection schemes. Further, they have considered a decoupled penalty-projection scheme in [15]. With the stabilization parameter tending to infinity, they have found that the solution of the decoupled penaltyprojection method converges to the associated solution of the fully decoupled method. Ashraf et al. [2] have considered the asymmetric flow of a micropolar fluid in a porous channel. They have transformed the governing equations of motion into ordinary differential equations by a similarity transformation and have investigated numerically the influence of an asymmetric parameter on the flow velocities and the microrotation. In order to describe a steady, laminar, incompressible, and two-dimensional flow of a micropolar fluid between two stationary porous coaxial disks, Kamal et al. [16] have used the micropolar fluid model and obtained a numerical solution by Berman's similarity transformation and the SOR iterative method.

The micropolar fluid model is a system of parabolic partial differential equations coupling the linear velocity and the pressure with the angular velocity: material particles have both translational and rotational degrees of freedom [26]. The overall goal of this work is to develop and investigate a decoupling finite element method with different time steps to solve this model effectively. For the strategy of different time steps, we refer to [8, 13, 17, 28, 35, 37] and the references therein. In particular, Connors and Howell [7] have presented a full discretization of the fluid-fluid interaction problem allowing different-sized time steps for the decoupled subcalculations. Shi et al. [38, 39] have designed a multistep technique to overcome the instability mainly caused by the explicit treatment of the convection system and to enlarge the stability region such that the resulting scheme behaves like an unconditionally stable scheme. Shan et al. [36] have analyzed a multirate and decoupling algorithm for the coupled time-dependent Stokes equations with Darcy's law, which allows different time steps in the fluid region and the porous region. Later, as an extension of the decoupling algorithm for the Stokes-Darcy system, the second-order backward differentiation formula [20], the second-order spectral deferred correction method [41], and an explicit leap frog method [19] have been designed. Besides that, the differing time steps method has been extended to the Navier-Stokes/Darcy model [14], the Darcy-Brinkman problem [22], and to the nematic liquid crystal flow [21]. 
In this paper, we study a decoupled finite element method with different time steps for the different physical fields to solve the micropolar fluid model. The remainder of this paper is organized as follows. In Section 2, we introduce the governing equations of the micropolar fluid model and some mathematical preliminaries. Then in the next section, a decoupling finite element method with different time steps is described for these equations, and its stability is analysed. An error analysis of the proposed algorithm is provided in Section 4. In Section 5, some numerical experiments are carried out to verify the theoretical analysis of the presented algorithm. Section 6 is the conclusion of this paper.

2. Notation and preliminaries. This work is concerned with the following governing equations of the micropolar fluid model $[9,25]$ :

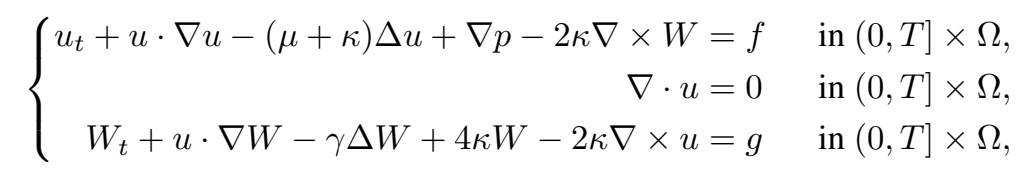

where $\Omega$ is a bounded domain in $\mathbb{R}^{2}$ and $T \in(0, \infty)$ is the final time, together with the following homogeneous boundary and initial conditions:

$$
\left\{\begin{aligned}
\left.u\right|_{S_{T}} & =0, & \left.W\right|_{S_{T}} & =0, \\
u(x, 0) & =u_{0}(x), & W(x, 0) & =W_{0}(x),
\end{aligned}\right.
$$

where $S_{T}:=\partial \Omega \times[0, T]$. Moreover, $u=\left(u_{1}, u_{2}, 0\right), W=\left(0,0, W_{3}\right)$, and $p$ represent the linear velocity, the angular velocity of the rotation of particles, and the pressure of the fluid, respectively. The three nondimensional numbers appearing in (2.1) are the Newtonian kinematic viscosity $\mu$, the dynamic micro-rotation viscosity $\kappa$, and the angular viscosity $\gamma$ [24]. Furthermore, $f=\left(f_{1}, f_{2}, 0\right)$ and $g=\left(0,0, g_{3}\right)$ represent smooth, externally applied forces and moments [33], respectively.

In view of this model, when the microgyration effects are neglected or when $W=0$, the micropolar fluid flow reduces to the Navier-Stokes flow. Hence, (2.1) can be regarded as a modification of the Navier-Stokes equations, or vice versa, the Navier-Stokes equations are viewed as characterizing the flow of a simplified micropolar fluid.

We use the standard notation for the Lebesgue space $L^{p}(\Omega)$ and the Sobolev spaces $W^{n, q}(\Omega)$, for $1 \leq q \leq \infty$ and $n \in \mathbb{N}^{+}$. The $L^{p}(\Omega)$-norms for $p \neq 2$ are denoted as $\|\cdot\|_{L^{p}}$, and the $L^{2}(\Omega)$-norm is denoted by $\|\cdot\|_{0}$. Moreover, the $W^{n, 2}(\Omega)=H^{n}(\Omega)$-norms are denoted by $\|\cdot\|_{n}$. Furthermore, define the dual space of $H^{1}(\Omega)$ as $H^{-1}(\Omega)$ with its norm given by

$$
\|f\|_{-1}=\sup _{v \in H^{1}(\Omega)} \frac{|(f, v)|}{\|\nabla v\|_{0}} .
$$

Throughout the paper, we also need the following function spaces:

$$
\begin{aligned}
& X=H_{0}^{1}(\Omega)^{2}=\left\{v \in H^{1}(\Omega)^{2}:\left.v\right|_{\partial \Omega}=0\right\}, \quad Q=\left\{q \in L^{2}(\Omega):(q, 1)=0\right\}, \\
& M=\left\{\phi \in H^{1}(\Omega):\left.\phi\right|_{\partial \Omega}=0\right\} .
\end{aligned}
$$

Then, based on the above definitions, we have the following variational formulation of problem (2.1)-(2.2): 
Find $(u, p, W) \in L^{2}(0, T ; X) \times L^{2}(0, T ; Q) \times L^{2}(0, T ; M)$ such that for all $t \in(0, T]$ and $(v, q, \phi) \in X \times Q \times M$,

$$
\begin{aligned}
\left(u_{t}, v\right)+b(u, u, v)+(\mu+\kappa)(\nabla u, \nabla v)-(p, \nabla \cdot v)-2 \kappa(\nabla \times W, v) & =(f, v), \\
(\nabla \cdot u, q) & =0, \\
\left(W_{t}, \phi\right)+b(u, W, \phi)+\gamma(\nabla W, \nabla \phi)+4 \kappa(W, \phi)-2 \kappa(\nabla \times u, \phi) & =(g, \phi),
\end{aligned}
$$

where

$$
\begin{aligned}
b(u, v, w) & =(u \cdot \nabla v, w)+((\nabla \cdot u) v, w) \\
& =\frac{1}{2}(u \cdot \nabla v, w)-\frac{1}{2}(u \cdot \nabla v, w), \quad \forall u, v, w \in H_{0}^{1}(\Omega) .
\end{aligned}
$$

The form $b$ satisfies the following properties [26]:

$$
\begin{gathered}
b(u, v, v)=0, \quad b(u, v, w) \leq c\|u\|_{1}\|v\|_{1}\|w\|_{1}, \quad \forall u, v, w \in H_{0}^{1}(\Omega), \\
b(u, v, w) \leq c\|u\|_{0}\|v\|_{2}\|w\|_{1}, \quad \forall u \in L^{2}(\Omega), v \in H^{2}(\Omega), w \in H_{0}^{1}(\Omega) .
\end{gathered}
$$

Here and in the sequel, we denote by $c$ (with or without a subscript) a general positive constant independent of the mesh size which may stand for different values at different occurrences.

3. A decoupling finite element method with different time steps. In this section, we construct a decoupling finite element method with different time steps for the different physical fields of the micropolar fluid model. We consider the situation that the linear velocity has a faster dynamics than the angular velocity.

From now on, $\mathcal{T}_{h}$ is a uniform partition of the domain $\Omega$ into triangular element $K$ with diameters bounded by a real positive parameter $h=\max _{K \in \mathcal{T}_{h}}\{\operatorname{diam}(K)\}$. Further, we introduce the finite element subspace $X_{h} \subset X, Q_{h} \subset Q$, and $M_{h} \subset M$ as follows:

$$
\begin{aligned}
& X_{h}=\left\{v_{h} \in X \cap C^{0}(\bar{\Omega})^{2} ;\left.v_{h}\right|_{K} \in P_{s}(K)^{2}, \forall K \in \mathcal{T}_{h}\right\}, \\
& Q_{h}=\left\{q_{h} \in Q \cap C^{0}(\bar{\Omega}) ;\left.q_{h}\right|_{K} \in P_{i}(K), \forall K \in \mathcal{T}_{h}\right\}, \\
& M_{h}=\left\{\phi_{h} \in M \cap C^{0}(\bar{\Omega}) ;\left.\phi_{h}\right|_{K} \in P_{j}(K), \forall K \in \mathcal{T}_{h}\right\},
\end{aligned}
$$

where $P_{l}(K)$ is the space of piecewise polynomials of degree $l \geq 1$ on $K$ and $l=s, i, j$. Assume that a pair of finite element spaces $\left(X_{h}, Q_{h}\right)$ satisfies the discrete LBB condition, i.e., there is a constant $\beta$ independent of the mesh size $h$ such that

$$
\sup _{v_{h} \in X_{h}} \frac{\left(q_{h}, \nabla \cdot v_{h}\right)}{\left\|\nabla v_{h}\right\|_{0}} \geq \beta\left\|q_{h}\right\|_{0}, \quad \forall q_{h} \in Q_{h} .
$$

Following [26, 33, 36], for $t \in[0, T]$, we define a projection operator

$$
\begin{aligned}
P_{h}: L^{2}(0, T ; X) \times L^{2}(0, T ; Q) \times L^{2}(0, T ; M) & \rightarrow X_{h} \times Q_{h} \times M_{h} \\
(u(t), p(t), W(t)) & \rightarrow\left(P_{h}^{u} u(t), P_{h}^{p} p(t), P_{h}^{W} W(t)\right)
\end{aligned}
$$

by

$$
\begin{aligned}
(\mu+\kappa)\left(\nabla P_{h}^{u} u(t), \nabla v_{h}\right) & -\left(P_{h}^{p} p(t), \nabla \cdot v_{h}\right) & & \\
& =(\mu+\kappa)\left(\nabla u(t), \nabla v_{h}\right)-\left(p(t), \nabla \cdot v_{h}\right), & & \forall v_{h} \in X_{h}, \\
\left(\nabla \cdot P_{h}^{u} u(t), q_{h}\right) & =\left(\nabla \cdot u(t), q_{h}\right), & & \forall q_{h} \in Q_{h}, \\
\gamma\left(\nabla P_{h}^{W} W(t), \nabla \phi_{h}\right)+4 & \kappa\left(P_{h}^{W} W(t), \phi_{h}\right) & & \\
& =\gamma\left(\nabla W(t), \nabla \phi_{h}\right)+4 \kappa\left(W(t), \phi_{h}\right), & & \forall \phi_{h} \in M_{h},
\end{aligned}
$$


which has the following approximation properties:

$$
\begin{aligned}
\left\|P_{h}^{u} u(t)-u(t)\right\|_{0}+h \| & P_{h}^{u} u(t)-u(t) \|_{1} & & \\
& \leq c h^{k+1}\|u(t)\|_{k+1}, & & \forall u \in X \cap H^{k+1}(\Omega)^{2}, \\
\left\|P_{h}^{p} p(t)-p(t)\right\|_{0} & \leq c h^{k}\|p(t)\|_{k}, & & \forall p \in Q \cap H^{k}(\Omega), \\
\left\|P_{h}^{W} W(t)-W(t)\right\|_{0}+h \| & P_{h}^{W} W(t)-W(t) \|_{1} & & \\
& \leq c h^{k+1}\|W(t)\|_{k+1}, & & \forall W \in M \cap H^{k+1}(\Omega),
\end{aligned}
$$

where $1 \leq k \leq l$ is an integer.

Furthermore, we assume that for each time level $s_{j}$, for the angular velocity there exists a time level $t_{m_{j}}$. For convenience, we further assume uniform time levels, that is,

$$
s_{j}=j \Delta s, j=0,1, \ldots, \bar{M}, \quad \Delta s=r \Delta t, \quad \text { and } \quad t_{m}=m \Delta t, m=0,1, \ldots, N,
$$

where $\Delta t=\frac{T}{N}$ and $N=r \bar{M}, m_{j}=j r$. Here, $r \geq 1$ is the key to the strategy of different time steps. If $r=1$, then the scheme becomes the classical time discretization method.

Let us now describe the algorithm: For $t_{m}$ and $t_{m_{j}} \in[0, T]$, the tuple $\left(u_{h}^{m}, p_{h}^{m}, W_{h}^{m_{j}}\right)$ denotes the discrete approximation of $\left(u\left(t_{m}\right), p\left(t_{m}\right), W\left(t_{m_{j}}\right)\right)$.

ALGORITHM 1.

Step I: Find $\left(u_{h}^{m+1}, p_{h}^{m+1}\right) \in X_{h} \times Q_{h}$, with $m=m_{j}, m_{j}+1, \ldots, m_{j+1}-1$, such that for all $\left(v_{h}, q_{h}\right) \in X_{h} \times Q_{h}$,

$$
\begin{aligned}
&\left(\frac{u_{h}^{m+1}-u_{h}^{m}}{\Delta t}, v_{h}\right)+b\left(u_{h}^{m}, u_{h}^{m+1}, v_{h}\right) \\
&+(\mu+\kappa)\left(\nabla u_{h}^{m+1}, \nabla v_{h}\right)-\left(p_{h}^{m+1}, \nabla \cdot v_{h}\right) \\
&-2 \kappa\left(\nabla \times W_{h}^{m_{j}}, v_{h}\right)+\left(q_{h}, \nabla \cdot u_{h}^{m+1}\right)=\left(f^{m+1}, v_{h}\right), \\
& u_{h}^{0}=P_{h}^{u} u_{0},
\end{aligned}
$$

with a small time step size $\Delta t$.

Step II: Set $S^{m_{j}}=\frac{1}{r} \sum_{i=m_{j}}^{m_{j+1}-1} u_{h}^{i}$.

Step III: Find $W_{h}^{m_{j+1}} \in M_{h}$ such that for all $\phi_{h} \in M_{h}$,

$$
\begin{aligned}
\left(\frac{W_{h}^{m_{j+1}}-W_{h}^{m_{j}}}{\Delta s}, \phi_{h}\right)+b\left(S^{m_{j}}, W_{h}^{m_{j+1}}, \phi_{h}\right) & \\
+\gamma\left(\nabla W_{h}^{m_{j+1}}, \nabla \phi_{h}\right)+4 \kappa\left(W_{h}^{m_{j+1}}, \phi_{h}\right)-2 \kappa\left(\nabla \times S^{m_{j}}, \phi_{h}\right) & =\left(g^{m_{j+1}}, \phi_{h}\right) \\
W_{h}^{0} & =P_{h}^{W} W_{0}
\end{aligned}
$$

with a large time step size $\Delta s=r \Delta t$.

Step IV: Set $j=j+1$, and repeat until $j=\bar{M}-1$.

We state the following theorem, which provides the stability of Algorithm 1.

THEOREM 3.1. The linear velocity $u_{h}^{m}$ in the first large time interval $\left[0, s_{1}\right]$ is stable for any $0 \leq J \leq r-2$, and the following a priori estimate is valid:

$$
\begin{aligned}
\left\|u_{h}^{J+1}\right\|_{0}^{2}+\sum_{i=0}^{J}\left\|u_{h}^{i+1}-u_{h}^{i}\right\|_{0}^{2} & +\Delta t \mu \sum_{i=0}^{J}\left\|\nabla u_{h}^{i+1}\right\|_{0}^{2} \\
& \leq\left\|u_{h}^{0}\right\|_{0}^{2}+2 \Delta t \kappa\left\|W_{h}^{0}\right\|_{0}^{2}+\frac{\Delta t}{\mu} \sum_{i=0}^{J}\left\|f^{i+1}\right\|_{-1}^{2},
\end{aligned}
$$


Moreover, the linear velocity $u_{h}^{m}$ and the angular velocity $W_{h}^{m_{j}}$ are stable for all $0 \leq l \leq \bar{M}-$ 1 and $-1 \leq J \leq r-2$, and the following a priori estimate

$$
\begin{aligned}
& \left\|u_{h}^{m_{l+1}+J+1}\right\|_{0}^{2}+\sum_{i=0}^{m_{l+1}+J}\left\|u_{h}^{i+1}-u_{h}^{i}\right\|_{0}^{2}+\Delta t \mu \sum_{i=0}^{m_{l+1}+J}\left\|\nabla u_{h}^{i+1}\right\|_{0}^{2}+\left\|W_{h}^{m_{l+1}}\right\|_{0}^{2} \\
& +\sum_{j=0}^{l}\left\|W_{h}^{m_{j+1}}-W_{h}^{m_{j}}\right\|_{0}^{2}+r \Delta t \gamma \sum_{j=0}^{l}\left\|\nabla W_{h}^{m_{j+1}}\right\|_{0}^{2}+8 r \Delta t \kappa \sum_{j=0}^{l}\left\|W_{h}^{m_{j+1}}\right\|_{0}^{2} \\
& \quad \leq \exp \left(2 C_{\dagger} T\right)\left(\left\|u_{h}^{0}\right\|_{0}^{2}+\left\|W_{h}^{0}\right\|_{0}^{2}+\frac{\Delta t}{\mu} \sum_{i=0}^{m_{l+1}+J}\left\|f^{i+1}\right\|_{-1}^{2}+\frac{2 r \Delta t}{\gamma} \sum_{j=0}^{l}\left\|g^{m_{j+1}}\right\|_{-1}^{2}\right),
\end{aligned}
$$

is valid when $C_{\dagger} \Delta t<\frac{1}{2}$ with $C_{\dagger}=\max \left\{2 r \kappa, 8 \kappa^{2}(r \gamma)^{-1}\right\}$.

Proof. Let $\left(v_{h}, q_{h}\right)=2 \Delta t\left(u_{h}^{m+1}, p_{h}^{m+1}\right)$ in (3.3) and use (2.4). Then,

$$
\begin{aligned}
\left(u_{h}^{m+1}-u_{h}^{m}, 2 u_{h}^{m+1}\right)+2 \Delta t(\mu+\kappa)\left(\nabla u_{h}^{m+1}, \nabla u_{h}^{m+1}\right) & -4 \Delta t \kappa\left(\nabla \times W_{h}^{m_{j}}, u_{h}^{m+1}\right) \\
& =2 \Delta t\left(f^{m+1}, u_{h}^{m+1}\right)
\end{aligned}
$$

Applying the polarization identity and summing the resulting equation over $m=m_{j}, m_{j}+$ $1, \ldots, m_{j+1}-1$ yield

$$
\begin{array}{r}
\left\|u_{h}^{m_{j+1}}\right\|_{0}^{2}-\left\|u_{h}^{m_{j}}\right\|_{0}^{2}+\sum_{i=m_{j}}^{m_{j+1}-1}\left\|u_{h}^{i+1}-u_{h}^{i}\right\|_{0}^{2}+2 \Delta t(\mu+\kappa) \sum_{i=m_{j}}^{m_{j+1}-1}\left\|\nabla u_{h}^{i+1}\right\|_{0}^{2} \\
=4 \Delta t \kappa \sum_{i=m_{j}}^{m_{j+1}-1}\left(\nabla \times W_{h}^{m_{j}}, u_{h}^{i+1}\right)+2 \Delta t \sum_{i=m_{j}}^{m_{j+1}-1}\left(f^{i+1}, u_{h}^{i+1}\right) .
\end{array}
$$

Moreover, choose $\phi_{h}=2 \Delta s W_{h}^{m_{j+1}}=2 r \Delta t W_{h}^{m_{j+1}}$ in (3.4) and use the polarization identity and (2.4) again to conclude that

$$
\begin{aligned}
& \left\|W_{h}^{m_{j+1}}\right\|_{0}^{2}-\left\|W_{h}^{m_{j}}\right\|_{0}^{2}+\left\|W_{h}^{m_{j+1}}-W_{h}^{m_{j}}\right\|_{0}^{2} \\
& +2 r \Delta t \gamma\left\|\nabla W_{h}^{m_{j+1}}\right\|_{0}^{2}+8 r \Delta t \kappa\left\|W_{h}^{m_{j+1}}\right\|_{0}^{2} \\
& \quad=4 r \Delta t \kappa\left(\nabla \times S^{m_{j}}, W_{h}^{m_{j+1}}\right)+2 r \Delta t\left(g^{m_{j+1}}, W_{h}^{m_{j+1}}\right) .
\end{aligned}
$$

Now, by using the Cauchy-Schwarz inequality and Young's inequality, we deduce

$$
\begin{aligned}
& 4 \Delta t \kappa \sum_{i=m_{j}}^{m_{j+1}-1}\left(\nabla \times W_{h}^{m_{j}}, u_{h}^{i+1}\right) \quad+4 r \Delta t \kappa\left(\nabla \times S^{m_{j}}, W_{h}^{m_{j+1}}\right) \\
& +2 \Delta t \sum_{i=m_{j}}^{m_{j+1}-1}\left(f^{i+1}, u_{h}^{i+1}\right)+2 r \Delta t\left(g^{m_{j+1}}, W_{h}^{m_{j+1}}\right) \\
& \leq 2 r \Delta t \kappa\left\|W_{h}^{m_{j}}\right\|_{0}^{2}+\Delta t(2 \kappa+\mu) \sum_{i=m_{j}}^{m_{j+1}-1}\left\|\nabla u_{h}^{i+1}\right\|_{0}^{2}+\frac{\Delta t}{\mu} \sum_{i=m_{j}}^{m_{j+1}-1}\left\|f^{i+1}\right\|_{-1}^{2} \\
& \quad+\frac{8 \Delta t \kappa^{2}}{r \gamma} \sum_{i=m_{j}}^{m_{j+1}-1}\left\|u_{h}^{i}\right\|_{0}^{2}+r \Delta t \gamma\left\|\nabla W_{h}^{m_{j+1}}\right\|_{0}^{2}+\frac{2 r \Delta t}{\gamma}\left\|g^{m_{j+1}}\right\|_{-1}^{2},
\end{aligned}
$$


where we have used the fact that

$$
(a, \nabla \times b)=(\nabla \times a, b) \quad \text { for } a, b \in H_{0}^{1}(\Omega) \quad \text { and } \quad\|\nabla a\|_{0}^{2}=\|\nabla \times a\|_{0}^{2}+\|\nabla \cdot a\|_{0}^{2} .
$$

Then, combining (3.7), (3.8), and (3.9), we get

$$
\begin{gathered}
\left\|u_{h}^{m_{j+1}}\right\|_{0}^{2}-\left\|u_{h}^{m_{j}}\right\|_{0}^{2}+\sum_{i=m_{j}}^{m_{j+1}-1}\left\|u_{h}^{i+1}-u_{h}^{i}\right\|_{0}^{2}+\Delta t \mu \sum_{i=m_{j}}^{m_{j+1}-1}\left\|\nabla u_{h}^{i+1}\right\|_{0}^{2} \\
+\left\|W_{h}^{m_{j+1}}\right\|_{0}^{2}-\left\|W_{h}^{m_{j}}\right\|_{0}^{2}+\left\|W_{h}^{m_{j+1}}-W_{h}^{m_{j}}\right\|_{0}^{2} \\
+r \Delta t \gamma\left\|\nabla W_{h}^{m_{j+1}}\right\|_{0}^{2}+8 r \Delta t \kappa\left\|W_{h}^{m_{j+1}}\right\|_{0}^{2} \\
\leq 2 r \Delta t \kappa\left\|W_{h}^{m_{j}}\right\|_{0}^{2}+\frac{8 \Delta t \kappa^{2}}{r \gamma} \sum_{i=m_{j}}^{m_{j+1}-1}\left\|u_{h}^{i}\right\|_{0}^{2} \\
+\frac{\Delta t}{\mu} \sum_{i=m_{j}}^{m_{j+1}-1}\left\|f^{i+1}\right\|_{-1}^{2}+\frac{2 r \Delta t}{\gamma}\left\|g^{m_{j+1}}\right\|_{-1}^{2} .
\end{gathered}
$$

Furthermore, summing (3.10) over all $j$ from 0 to $l, 0 \leq l \leq \bar{M}-1$, we obtain

$$
\begin{aligned}
& \left\|u_{h}^{m_{l+1}}\right\|_{0}^{2}+\sum_{j=0}^{l} \sum_{i=m_{j}}^{m_{j+1}-1}\left\|u_{h}^{i+1}-u_{h}^{i}\right\|_{0}^{2}+\Delta t \mu \sum_{j=0}^{l} \sum_{i=m_{j}}^{m_{j+1}-1}\left\|\nabla u_{h}^{i+1}\right\|_{0}^{2} \\
& +\left\|W_{h}^{m_{l+1}}\right\|_{0}^{2}+\sum_{j=0}^{l}\left\|W_{h}^{m_{j+1}}-W_{h}^{m_{j}}\right\|_{0}^{2} \\
& +r \Delta t \gamma \sum_{j=0}^{l}\left\|\nabla W_{h}^{m_{j+1}}\right\|_{0}^{2}+8 r \Delta t \kappa \sum_{j=0}^{l}\left\|W_{h}^{m_{j+1}}\right\|_{0}^{2} \\
& \leq\left\|u_{h}^{0}\right\|_{0}^{2}+\left\|W_{h}^{0}\right\|_{0}^{2}+2 r \Delta t \kappa \sum_{j=0}^{l}\left\|W_{h}^{m_{j}}\right\|_{0}^{2}+\frac{8 \Delta t \kappa^{2}}{r \gamma} \sum_{j=0}^{l} \sum_{i=m_{j}}^{m_{j+1}-1}\left\|u_{h}^{i}\right\|_{0}^{2} \\
& \quad+\frac{\Delta t}{\mu} \sum_{j=0}^{l} \sum_{i=m_{j}}^{m_{j+1}-1}\left\|f^{i+1}\right\|_{-1}^{2}+\frac{2 r \Delta t}{\gamma} \sum_{j=0}^{l}\left\|g^{m_{j+1}}\right\|_{-1}^{2} .
\end{aligned}
$$

Summing (3.6) over $m=m_{l+1}, m_{l+1}+1, \ldots, m_{l+1}+J, 0 \leq J \leq r-2$ and using the Cauchy-Schwarz inequality and Young's inequality, we also arrive at

$$
\begin{gathered}
\left\|u_{h}^{m_{l+1}+J+1}\right\|_{0}^{2}-\left\|u_{h}^{m_{l+1}}\right\|_{0}^{2}+\sum_{i=m_{l+1}}^{m_{l+1}+J}\left\|u_{h}^{i+1}-u_{h}^{i}\right\|_{0}^{2}+2 \Delta t(\mu+\kappa) \sum_{i=m_{l+1}}^{m_{l+1}+J}\left\|\nabla u_{h}^{i+1}\right\|_{0}^{2} \\
=4 \Delta t \kappa \sum_{i=m_{l+1}}^{m_{l+1}+J}\left(\nabla \times W_{h}^{m_{l+1}}, u_{h}^{i+1}\right)+2 \Delta t \sum_{i=m_{l+1}}^{m_{l+1}+J}\left(f^{i+1}, u_{h}^{i+1}\right) \\
\leq 2 \Delta t \kappa \sum_{i=m_{l+1}}^{m_{l+1}+J}\left\|\nabla u_{h}^{i+1}\right\|_{0}^{2}+2 r \Delta t \kappa\left\|W_{h}^{m_{l+1}}\right\|_{0}^{2} \\
+\frac{\Delta t}{\mu} \sum_{i=m_{l+1}}^{m_{l+1}+J}\left\|f^{i+1}\right\|_{-1}^{2}+\Delta t \mu \sum_{i=m_{l+1}}^{m_{l+1}+J}\left\|\nabla u_{h}^{i+1}\right\|_{0}^{2} .
\end{gathered}
$$


Hence, it follows that

$$
\begin{gathered}
\left\|u_{h}^{m_{l+1}+J+1}\right\|_{0}^{2}-\left\|u_{h}^{m_{l+1}}\right\|_{0}^{2}+\sum_{i=m_{l+1}}^{m_{l+1}+J}\left\|u_{h}^{i+1}-u_{h}^{i}\right\|_{0}^{2}+\Delta t \mu \sum_{i=m_{l+1}}^{m_{l+1}+J}\left\|\nabla u_{h}^{i+1}\right\|_{0}^{2} \\
\leq 2 r \Delta t \kappa\left\|W_{h}^{m_{l+1}}\right\|_{0}^{2}+\frac{\Delta t}{\mu} \sum_{i=m_{l+1}}^{m_{l+1}+J}\left\|f^{i+1}\right\|_{-1}^{2} .
\end{gathered}
$$

On the one hand, notice that (3.12) with $l=-1$ can be rewritten as

$$
\begin{aligned}
\left\|u_{h}^{J+1}\right\|_{0}^{2}+\sum_{i=0}^{J}\left\|u_{h}^{i+1}-u_{h}^{i}\right\|_{0}^{2}+\Delta t \mu \sum_{i=0}^{J}\left\|\nabla u_{h}^{i+1}\right\|_{0}^{2} \\
\leq\left\|u_{h}^{0}\right\|_{0}^{2}+2 r \Delta t \kappa\left\|W_{h}^{0}\right\|_{0}^{2}+\frac{\Delta t}{\mu} \sum_{i=0}^{J}\left\|f^{i+1}\right\|_{-1}^{2},
\end{aligned}
$$

which is (3.5). On the other hand, combining (3.11) with (3.12), we obtain

$$
\begin{gathered}
\left\|u_{h}^{m_{l+1}+J+1}\right\|_{0}^{2}+\sum_{i=0}^{m_{l+1}+J}\left\|u_{h}^{i+1}-u_{h}^{i}\right\|_{0}^{2}+\Delta t \mu \sum_{i=0}^{m_{l+1}+J}\left\|\nabla u_{h}^{i+1}\right\|_{0}^{2}+\left\|W_{h}^{m_{l+1}}\right\|_{0}^{2} \\
+\sum_{j=0}^{l}\left\|W_{h}^{m_{j+1}}-W_{h}^{m_{j}}\right\|_{0}^{2}+r \Delta t \gamma \sum_{j=0}^{l}\left\|\nabla W_{h}^{m_{j+1}}\right\|_{0}^{2}+8 r \Delta t \kappa \sum_{j=0}^{l}\left\|W_{h}^{m_{j+1}}\right\|_{0}^{2} \\
\leq\left\|u_{h}^{0}\right\|_{0}^{2}+\left\|W_{h}^{0}\right\|_{0}^{2}+2 r \Delta t \kappa \sum_{j=0}^{l+1}\left\|W_{h}^{m_{j}}\right\|_{0}^{2}+\frac{8 \Delta t \kappa^{2}}{r \gamma} \sum_{j=0}^{l} \sum_{i=m_{j}}^{m_{j+1}+J+1}\left\|u_{h}^{i}\right\|_{0}^{2} \\
+\frac{\Delta t}{\mu} \sum_{i=0}^{m_{l+1}+J}\left\|f^{i+1}\right\|_{-1}^{2}+\frac{2 r \Delta t}{\gamma} \sum_{j=0}^{l}\left\|g^{m_{j+1}}\right\|_{-1}^{2} .
\end{gathered}
$$

Denote $C_{\dagger}=\max \left\{2 r \kappa, 8 \kappa^{2}(r \gamma)^{-1}\right\}$ and choose $\Delta t$ such that it satisfies $C_{\dagger} \Delta t<\frac{1}{2}$. By the discrete Grönwall inequality, we get the stability estimate

$$
\begin{aligned}
& \left\|u_{h}^{m_{l+1}+J+1}\right\|_{0}^{2}+\sum_{i=0}^{m_{l+1}+J}\left\|u_{h}^{i+1}-u_{h}^{i}\right\|_{0}^{2}+\Delta t \mu \sum_{i=0}^{m_{l+1}+J}\left\|\nabla u_{h}^{i+1}\right\|_{0}^{2}+\left\|W_{h}^{m_{l+1}}\right\|_{0}^{2} \\
& +\sum_{j=0}^{l}\left\|W_{h}^{m_{j+1}}-W_{h}^{m_{j}}\right\|_{0}^{2}+r \Delta t \gamma \sum_{j=0}^{l}\left\|\nabla W_{h}^{m_{j+1}}\right\|_{0}^{2}+8 r \Delta t \kappa \sum_{j=0}^{l}\left\|W_{h}^{m_{j+1}}\right\|_{0}^{2} \\
& \quad \leq \exp \left(2 C_{\dagger} T\right)\left(\left\|u_{h}^{0}\right\|_{0}^{2}+\left\|W_{h}^{0}\right\|_{0}^{2}+\frac{\Delta t}{\mu} \sum_{i=0}^{m_{l+1}+J}\left\|f^{i+1}\right\|_{-1}^{2}+\frac{2 r \Delta t}{\gamma} \sum_{j=0}^{l}\left\|g^{m_{j+1}}\right\|_{-1}^{2}\right) .
\end{aligned}
$$

(

4. Error estimates for the algorithm. In this section, we present the convergence analysis for Algorithm 1 and derive an a priori error estimate for the fields. In order to get the error of Algorithm 1, we at first state some notation. Let

$$
\tilde{u}^{m}=P_{h}^{u} u\left(t_{m}\right), \quad \tilde{p}^{m}=P_{h}^{p} p\left(t_{m}\right), \quad \text { and } \quad \tilde{W}^{m}=P_{h}^{W} W\left(t_{m}\right) .
$$


Set

$$
\begin{array}{lll}
e_{u}^{m}=u\left(t_{m}\right)-\tilde{u}^{m}, & \epsilon_{p}^{m}=p\left(t_{m}\right)-\tilde{p}^{m}, & \xi_{W}^{m}=W\left(t_{m}\right)-\tilde{W}^{m}, \\
e^{m}=\tilde{u}^{m}-u_{h}^{m}, & \epsilon^{m}=\tilde{p}^{m}-p_{h}^{m}, & \xi^{m}=\tilde{W}^{m}-W_{h}^{m} .
\end{array}
$$

Furthermore, we assume that $e^{0}=0, \epsilon^{0}=0$, and $\xi^{0}=0$.

Next, we define

$$
\begin{aligned}
\Pi_{u, t}^{m+1} & =\frac{\tilde{u}^{m+1}-\tilde{u}^{m}}{\Delta t}-u_{t}\left(t_{m+1}\right) \\
& =\left(\frac{\tilde{u}^{m+1}-\tilde{u}^{m}}{\Delta t}-\frac{u\left(t_{m+1}\right)-u\left(t_{m}\right)}{\Delta t}\right)+\left(\frac{u\left(t_{m+1}\right)-u\left(t_{m}\right)}{\Delta t}-u_{t}\left(t_{m+1}\right)\right),
\end{aligned}
$$

and

$$
\begin{aligned}
\Pi_{W, s}^{m_{j+1}}= & \frac{\tilde{W}^{m_{j+1}}-\tilde{W}^{m_{j}}}{\Delta s}-W_{t}\left(t_{m_{j+1}}\right) \\
= & \left(\frac{\tilde{W}^{m_{j+1}}-\tilde{W}^{m_{j}}}{\Delta s}-\frac{W\left(t_{m_{j+1}}\right)-W\left(t_{m_{j}}\right)}{\Delta s}\right) \\
& \quad+\left(\frac{W\left(t_{m_{j+1}}\right)-W\left(t_{m_{j}}\right)}{\Delta s}-W_{t}\left(t_{m_{j+1}}\right)\right) .
\end{aligned}
$$

It is easy to verify that $\Pi_{u, t}^{m+1}$ and $\Pi_{W, s}^{m_{j+1}}$ satisfy the following bounds:

$$
\begin{aligned}
\left\|\Pi_{u, t}^{m+1}\right\|_{0}^{2} \leq & \frac{1}{\Delta t^{2}} \int_{\Omega}\left(\int_{t_{m}}^{t_{m+1}}\left(P_{h}^{u}-I\right) u_{t}(t) d t\right)^{2} d x \\
& \quad+\frac{1}{\Delta t^{2}} \int_{\Omega}\left(\int_{t_{m}}^{t_{m+1}}\left(t-t_{m}\right) u_{t t}(t) d t\right)^{2} d x \\
\leq & \frac{1}{\Delta t} \int_{t_{m}}^{t_{m+1}}\left\|\left(P_{h}^{u}-I\right) u_{t}(t)\right\|_{0}^{2} d t+\Delta t \int_{t_{m}}^{t_{m+1}}\left\|u_{t t}(t)\right\|_{0}^{2} d t
\end{aligned}
$$

and

$$
\left\|\Pi_{W, s}^{m_{j+1}}\right\|_{0}^{2} \leq \frac{1}{\Delta s} \int_{t_{m_{j}}}^{t_{m_{j+1}}}\left\|\left(P_{h}^{W}-I\right) W_{t}(t)\right\|_{0}^{2} d t+\Delta s \int_{t_{m_{j}}}^{t_{m_{j+1}}}\left\|W_{t t}(t)\right\|_{0}^{2} d t .
$$

One also finds that

$$
\begin{aligned}
\left\|\tilde{u}^{m+1}-\tilde{u}^{m}\right\|_{0}^{2} & =\left\|P_{h}^{u}\left(u\left(t_{m+1}\right)-u\left(t_{m}\right)\right)\right\|_{0}^{2} \leq c\left\|u\left(t_{m+1}\right)-u\left(t_{m}\right)\right\|_{0}^{2} \\
& \leq c \int_{\Omega}\left(\int_{t_{m}}^{t_{m+1}} u_{t}(t) d t\right)^{2} d x \leq c \Delta t \int_{t_{m}}^{t_{m+1}}\left\|u_{t}(t)\right\|_{0}^{2} d t,
\end{aligned}
$$

and

$$
\left\|\tilde{W}^{m+1}-\tilde{W}^{m}\right\|_{0}^{2} \leq c \Delta t \int_{t_{m}}^{t_{m+1}}\left\|W_{t}(t)\right\|_{0}^{2} d t
$$


According to (3.1) and (2.3), we have

$$
\begin{aligned}
\left(\frac{\tilde{u}^{m+1}-\tilde{u}^{m}}{\Delta t}, v_{h}\right)+(\mu+\kappa)\left(\nabla \tilde{u}^{m+1}, \nabla v_{h}\right) & \\
+b\left(u\left(t_{m+1}\right)\right. & \left., u\left(t_{m+1}\right), v_{h}\right) \\
-\left(\tilde{p}^{m+1}, \nabla \cdot\right. & \left.v_{h}\right)-2 \kappa\left(\nabla \times W\left(t_{m+1}\right), v_{h}\right) \\
& =\left(\Pi_{u, t}^{m+1}, v_{h}\right)+\left(f^{m+1}, v_{h}\right),
\end{aligned}
$$

$$
\begin{gathered}
\left(\nabla \cdot \tilde{u}^{m+1}, q_{h}\right)=0, \\
\left(\frac{\tilde{W}^{m_{j+1}}-\tilde{W}^{m_{j}}}{\Delta s}, \phi_{h}\right)+\gamma\left(\nabla \tilde{W}^{m_{j+1}}, \nabla \phi_{h}\right)+4 \kappa\left(\tilde{W}^{m_{j+1}}, \phi_{h}\right) \\
-2 \kappa\left(\nabla \times u\left(t_{m_{j+1}}\right), \phi_{h}\right)+b\left(u\left(t_{m_{j+1}}\right), W\left(t_{m_{j+1}}\right), \phi_{h}\right) \\
=\left(\Pi_{W, s}^{m_{j+1}}, \phi_{h}\right)+\left(g^{m_{j+1}}, \phi_{h}\right) .
\end{gathered}
$$

Then based on (4.3), (4.4), and (3.3), we obtain

$$
\begin{aligned}
& \left(\frac{e^{m+1}-e^{m}}{\Delta t}, v_{h}\right)+(\mu+\kappa)\left(\nabla e^{m+1}, \nabla v_{h}\right) \\
& \quad+b\left(u\left(t_{m+1}\right), u\left(t_{m+1}\right), v_{h}\right)-b\left(u_{h}^{m}, u_{h}^{m+1}, v_{h}\right) \\
& \quad-\left(\epsilon^{m+1}, \nabla \cdot v_{h}\right)+\left(\nabla \cdot e^{m+1}, q_{h}\right) \\
& \quad-2 \kappa\left(\nabla \times W\left(t_{m+1}\right), v_{h}\right)+2 \kappa\left(\nabla \times W_{h}^{m_{j}}, v_{h}\right)=\left(\Pi_{u, t}^{m+1}, v_{h}\right) .
\end{aligned}
$$

Besides that, subtract (4.5) from (3.4) to get

$$
\begin{gathered}
\left(\frac{\xi^{m_{j+1}}-\xi^{m_{j}}}{\Delta s}, \phi_{h}\right)+\gamma\left(\nabla \xi^{m_{j+1}}, \nabla \phi_{h}\right) \\
+4 \kappa\left(\xi^{m_{j+1}}, \phi_{h}\right)+b\left(u\left(t_{m_{j+1}}\right), W\left(t_{m_{j+1}}\right), \phi_{h}\right) \\
-b\left(S^{m_{j}}, W_{h}^{m_{j+1}}, \phi_{h}\right)-2 \kappa\left(\nabla \times u\left(t_{m_{j+1}}\right), \phi_{h}\right)+2 \kappa\left(\nabla \times S^{m_{j}}, \phi_{h}\right) \\
=\left(\Pi_{W, s}^{m_{j+1}}, \phi_{h}\right) .
\end{gathered}
$$

THEOREM 4.1. Suppose that the true solutions are smooth and that the time step $\Delta t$ satisfies

$$
4 \Delta t \kappa \leq 1, \quad\left(\frac{16 \kappa^{2}}{\gamma}+\frac{8 c^{* *}}{r \gamma}+\frac{8 c^{*}}{\mu}\right) \Delta t \leq 1 .
$$

Then the following estimate for the error at the large time step $\Delta s=r \Delta t$ holds:

$$
\begin{array}{r}
\left\|e^{m_{l+1}}\right\|_{0}^{2}+\Delta t(\mu+\kappa) \sum_{j=0}^{l} \sum_{i=m_{j}}^{m_{j+1}-1}\left\|\nabla e^{i+1}\right\|_{0}^{2}+\left\|\xi^{m_{l+1}}\right\|_{0}^{2} \\
+r \Delta t \gamma \sum_{j=0}^{l}\left\|\nabla \xi^{m_{j+1}}\right\|_{0}^{2}+8 r \Delta t \kappa \sum_{j=0}^{l}\left\|\xi^{m_{j+1}}\right\|_{0}^{2} \\
\leq c \Delta t^{2}+c h^{2(k+1)} .
\end{array}
$$


Proof. Setting $\left(v_{h}, q_{h}\right)=2 \Delta t\left(e^{m+1}, \epsilon^{m+1}\right)$ in (4.6) yields

$$
\begin{gathered}
\left(e^{m+1}-e^{m}, 2 e^{m+1}\right)+2 \Delta t(\mu+\kappa)\left(\nabla e^{m+1}, \nabla e^{m+1}\right) \\
+2 \Delta t b\left(u\left(t_{m+1}\right), u\left(t_{m+1}\right), e^{m+1}\right)-2 \Delta t b\left(u_{h}^{m}, u_{h}^{m+1}, e^{m+1}\right) \\
-4 \Delta t \kappa\left(\nabla \times W\left(t_{m+1}\right), e^{m+1}\right)+4 \Delta t \kappa\left(\nabla \times W_{h}^{m_{j}}, e^{m+1}\right) \\
=2 \Delta t\left(\Pi_{u, t}^{m+1}, e^{m+1}\right) .
\end{gathered}
$$

Then, summing (4.8) over $m=m_{j}, m_{j}+1, \ldots, m_{j+1}-1$, we obtain

$$
\begin{aligned}
\left\|e^{m_{j+1}}\right\|_{0}^{2}- & \left\|e^{m_{j}}\right\|_{0}^{2}+\sum_{i=m_{j}}^{m_{j+1}-1}\left\|e^{i+1}-e^{i}\right\|_{0}^{2}+2 \Delta t(\mu+\kappa) \sum_{i=m_{j}}^{m_{j+1}-1}\left\|\nabla e^{i+1}\right\|_{0}^{2} \\
=2 \Delta t & \sum_{i=m_{j}}^{m_{j+1}-1} b\left(u_{h}^{i}, u_{h}^{i+1}, e^{i+1}\right)-2 \Delta t \sum_{i=m_{j}}^{m_{j+1}-1} b\left(u\left(t_{i+1}\right), u\left(t_{i+1}\right), e^{i+1}\right) \\
& +4 \Delta t \kappa \sum_{i=m_{j}}^{m_{j+1}-1}\left(\nabla \times W\left(t_{i+1}\right), e^{i+1}\right)-4 \Delta t \kappa \sum_{i=m_{j}}^{m_{j+1}-1}\left(\nabla \times W_{h}^{m_{j}}, e^{i+1}\right) \\
& +2 \Delta t \sum_{i=m_{j}}^{m_{j+1}-1}\left(\Pi_{u, t}^{i+1}, e^{i+1}\right) .
\end{aligned}
$$

Then,

Let $\phi_{h}=2 \Delta s \xi^{m_{j+1}}=2 r \Delta t \xi^{m_{j+1}}$ in (4.7), use the polarization identity, and rearrange.

$$
\begin{aligned}
\left\|\xi^{m_{j+1}}\right\|_{0}^{2}-\left\|\xi^{m_{j}}\right\|_{0}^{2} & +\left\|\xi^{m_{j+1}}-\xi^{m_{j}}\right\|_{0}^{2}+2 r \Delta t \gamma\left\|\nabla \xi^{m_{j+1}}\right\|_{0}^{2}+8 r \Delta t \kappa\left\|\xi^{m_{j+1}}\right\|_{0}^{2} \\
=2 r \Delta t & \left.S^{m_{j}}, W_{h}^{m_{j+1}}, \xi^{m_{j+1}}\right)-2 r \Delta t b\left(u\left(t_{m_{j+1}}\right), W\left(t_{m_{j+1}}\right), \xi^{m_{j+1}}\right) \\
& +4 r \Delta t \kappa\left(\nabla \times u\left(t_{m_{j+1}}\right), \xi^{m_{j+1}}\right)-4 r \Delta t \kappa\left(\nabla \times S^{m_{j}}, \xi^{m_{j+1}}\right) \\
& +2 r \Delta t\left(\Pi_{W, s}^{m_{j+1}}, \xi^{m_{j+1}}\right)
\end{aligned}
$$

Furthermore, combining (4.9) and (4.10), we get

$$
\begin{aligned}
& \left\|e^{m_{j+1}}\right\|_{0}^{2}-\left\|e^{m_{j}}\right\|_{0}^{2}+\sum_{i=m_{j}}^{m_{j+1}-1}\left\|e^{i+1}-e^{i}\right\|_{0}^{2} \\
& +2 \Delta t(\mu+\kappa) \sum_{i=m_{j}}^{m_{j+1}-1}\left\|\nabla e^{i+1}\right\|_{0}^{2}+\left\|\xi^{m_{j+1}}\right\|_{0}^{2} \\
& -\left\|\xi^{m_{j}}\right\|_{0}^{2}+\left\|\xi^{m_{j+1}}-\xi^{m_{j}}\right\|_{0}^{2}+2 r \Delta t \gamma\left\|\nabla \xi^{m_{j+1}}\right\|_{0}^{2}+8 r \Delta t \kappa\left\|\xi^{m_{j+1}}\right\|_{0}^{2} \\
& =2 \Delta t \sum_{i=m_{j}}^{m_{j+1}-1} b\left(u_{h}^{i}, u_{h}^{i+1}, e^{i+1}\right)-2 \Delta t \sum_{i=m_{j}}^{m_{j+1}-1} b\left(u\left(t_{i+1}\right), u\left(t_{i+1}\right), e^{i+1}\right) \\
& +4 \Delta t \kappa \sum_{i=m_{j}}^{m_{j+1}-1}\left(\nabla \times W\left(t_{i+1}\right), e^{i+1}\right) \\
& -4 \Delta t \kappa \sum_{i=m_{j}}^{m_{j+1}-1}\left(\nabla \times W_{h}^{m_{j}}, e^{i+1}\right)
\end{aligned}
$$




$$
\begin{aligned}
& +2 \Delta t \sum_{i=m_{j}}^{m_{j+1}-1}\left(\Pi_{u, t}^{i+1}, e^{i+1}\right)+2 r \Delta t b\left(S^{m_{j}}, W_{h}^{m_{j+1}}, \xi^{m_{j+1}}\right) \\
& -2 r \Delta t b\left(u\left(t_{m_{j+1}}\right), W\left(t_{m_{j+1}}\right), \xi^{m_{j+1}}\right) \\
& +4 r \Delta t \kappa\left(\nabla \times u\left(t_{m_{j+1}}\right), \xi^{m_{j+1}}\right) \\
& -4 r \Delta t \kappa\left(\nabla \times S^{m_{j}}, \xi^{m_{j+1}}\right)+2 r \Delta t\left(\Pi_{W, s}^{m_{j+1}}, \xi^{m_{j+1}}\right):=\sum_{i=1}^{10} I_{i} .
\end{aligned}
$$

Now, we estimate each term of the right-hand side of the previous inequality. By the CauchySchwarz inequality and Young's inequality, we have

$$
\begin{aligned}
I_{5}+I_{10} \leq c \Delta t \sum_{i=m_{j}}^{m_{j+1}-1} & \left\|\Pi_{u, t}^{i+1}\right\|_{0}^{2}+c r \Delta t\left\|\Pi_{W, s}^{m_{j+1}}\right\|_{0}^{2} \\
& \quad+\frac{1}{2} \mu \Delta t \sum_{i=m_{j}}^{m_{j+1}-1}\left\|\nabla e^{i+1}\right\|_{0}^{2}+\frac{1}{4} r \Delta t \gamma\left\|\nabla \xi^{m_{j+1}}\right\|_{0}^{2},
\end{aligned}
$$

and

$$
\begin{gathered}
I_{3}+I_{4} \leq 4 \Delta t \kappa \sum_{i=m_{j}}^{m_{j+1}-1}\left\|W\left(t_{i+1}\right)-W_{h}^{m_{j}}\right\|_{0}^{2}+\Delta t \kappa \sum_{i=m_{j}}^{m_{j+1}-1}\left\|\nabla e^{i+1}\right\|_{0}^{2} \\
\leq 4 \Delta t \kappa \sum_{i=m_{j}}^{m_{j+1}-1}\left(\left\|W\left(t_{i+1}\right)-W\left(t_{i}\right)\right\|_{0}^{2}+\left\|W\left(t_{i}\right)-\tilde{W}^{i}\right\|_{0}^{2}\right. \\
\left.+\left\|\tilde{W}^{i}-\tilde{W}^{m_{j}}\right\|_{0}^{2}+\left\|\tilde{W}^{m_{j}}-W_{h}^{m_{j}}\right\|_{0}^{2}\right) \\
+\Delta t \kappa \sum_{i=m_{j}}^{m_{j+1}-1}\left\|\nabla e^{i+1}\right\|_{0}^{2} .
\end{gathered}
$$

In fact, one has

$$
\begin{gathered}
\left\|W\left(t_{i+1}\right)-W\left(t_{i}\right)\right\|_{0}^{2} \leq \Delta t \int_{t_{i}}^{t_{i+1}}\left\|W_{t}(t)\right\|_{0}^{2} d t \\
\left\|W\left(t_{i}\right)-\tilde{W}^{i}\right\|_{0}^{2}=\left\|\xi_{W}^{i}\right\|_{0}^{2} \leq c h^{2(k+1)}, \\
\left\|\tilde{W}^{m_{j}}-W_{h}^{m_{j}}\right\|_{0}^{2}=\left\|\xi^{m_{j}}\right\|_{0}^{2} \leq\left\|\xi^{m_{j}}-\xi^{m_{j+1}}\right\|_{0}^{2}+\left\|\xi^{m_{j+1}}\right\|_{0}^{2} .
\end{gathered}
$$

Hence, (4.13) is rewritten as

$$
\begin{aligned}
I_{3}+I_{4} \leq & 4 \Delta t \kappa \sum_{i=m_{j}}^{m_{j+1}-1}\left(\left\|\xi^{m_{j}}-\xi^{m_{j+1}}\right\|_{0}^{2}+\left\|\xi^{m_{j+1}}\right\|_{0}^{2}+\left\|\tilde{W}^{i}-\tilde{W}^{m_{j}}\right\|_{0}^{2}\right) \\
& +c \Delta t^{2} \sum_{i=m_{j}}^{m_{j+1}-1} \int_{t_{i}}^{t_{i+1}}\left\|W_{t}(t)\right\|_{0}^{2} d t+c r \Delta t h^{2(k+1)} \\
& +\Delta t \kappa \sum_{i=m_{j}}^{m_{j+1}-1}\left\|\nabla e^{i+1}\right\|_{0}^{2} .
\end{aligned}
$$


Similarly,

$$
\begin{aligned}
I_{8}+I_{9} \leq & \frac{16 r \Delta t \kappa^{2}}{\gamma}\left\|u\left(t_{m_{j+1}}\right)-\frac{1}{r} \sum_{i=m_{j}}^{m_{j+1}-1} u_{h}^{i}\right\|_{0}^{2}+\frac{1}{4} r \Delta t \gamma\left\|\nabla \xi^{m_{j+1}}\right\|_{0}^{2} \\
\leq & \frac{16 r \Delta t \kappa^{2}}{\gamma}\left(\frac{1}{r} \sum_{i=m_{j}}^{m_{j+1}-1}\left\|\tilde{u}^{m_{j}}-\tilde{u}^{i}\right\|_{0}^{2}+\frac{1}{r} \sum_{i=m_{j}}^{m_{j+1}-1}\left\|e^{i+1}-e^{i}\right\|_{0}^{2}\right. \\
& \left.+\frac{1}{r} \sum_{i=m_{j}}^{m_{j+1}-1}\left\|e^{i+1}\right\|_{0}^{2}\right) \\
& +c r^{2} \Delta t^{2} \int_{t_{m_{j}}}^{t_{m_{j+1}}}\left\|u_{t}(t)\right\|_{0}^{2} d t+c r \Delta t h^{2(k+1)}+\frac{1}{4} r \Delta t \gamma\left\|\nabla \xi^{m_{j+1}}\right\|_{0}^{2} .
\end{aligned}
$$

Now, we estimate $I_{1}$ and $I_{2}$. By adding and subtracting some terms and applying (2.4), we have

$$
\begin{aligned}
I_{1}+I_{2} \leq 2 \Delta t & \sum_{i=m_{j}}^{m_{j+1}-1}\left|b\left(u_{h}^{i}-\tilde{u}^{i}, u_{h}^{i+1}, e^{i+1}\right)\right|+2 \Delta t \sum_{i=m_{j}}^{m_{j+1}-1}\left|b\left(\tilde{u}^{i}-u\left(t_{i}\right), u_{h}^{i+1}, e^{i+1}\right)\right| \\
& +2 \Delta t \sum_{i=m_{j}}^{m_{j+1}-1}\left|b\left(u\left(t_{i}\right)-u\left(t_{i+1}\right), u_{h}^{i+1}, e^{i+1}\right)\right| \\
& +2 \Delta t \sum_{i=m_{j}}^{m_{j+1}-1}\left|b\left(u\left(t_{i+1}\right), u_{h}^{i+1}-u\left(t_{i+1}\right), e^{i+1}\right)\right| \\
\leq 2 \Delta t & \sum_{i=m_{j}}^{m_{j+1}-1}\left|b\left(e^{i}, e_{u}^{i+1}, e^{i+1}\right)\right|+2 \Delta t \sum_{i=m_{j}}^{m_{j+1}-1}\left|b\left(e^{i}, u\left(t_{i+1}\right), e^{i+1}\right)\right| \\
& +2 \Delta t \sum_{i=m_{j}}^{m_{j+1}-1}\left|b\left(e_{u}^{i}, e_{u}^{i+1}, e^{i+1}\right)\right|+2 \Delta t \sum_{i=m_{j}}^{m_{j+1}-1}\left|b\left(e_{u}^{i}, u\left(t_{i+1}\right), e^{i+1}\right)\right| \\
& +2 \Delta t \sum_{i=m_{j}}^{m_{j+1}-1}\left|b\left(u\left(t_{i}\right)-u\left(t_{i+1}\right), u\left(t_{i+1}\right), e^{i+1}\right)\right| \\
& +2 \Delta t \sum_{i=m_{j}}^{m_{j+1}-1}\left|b\left(u\left(t_{i+1}\right), e_{u}^{i+1}, e^{i+1}\right)\right| \\
& +2 \Delta t \sum_{i=m_{j}}^{m_{j+1}-1}\left|b\left(u\left(t_{i}\right)-u\left(t_{i+1}\right), e_{u}^{i+1}, e^{i+1}\right)\right|:=\sum_{i=1}^{7} R_{i} .
\end{aligned}
$$

Further, by applying the Cauchy-Schwarz inequality and Young's inequality and (2.5), we arrive at

$$
\begin{aligned}
& R_{1}+R_{2} \leq \frac{8}{\mu} \Delta t \sum_{i=m_{j}}^{m_{j+1}-1}\left\|e^{i}\right\|_{0}^{2}\left(\left\|e_{u}^{i+1}\right\|_{2}^{2}+\left\|u\left(t_{i+1}\right)\right\|_{2}^{2}\right)+\frac{1}{8} \mu \Delta t \sum_{i=m_{j}}^{m_{j+1}-1}\left\|\nabla e^{i+1}\right\|_{0}^{2}, \\
& R_{3}+R_{4} \leq c \Delta t \sum_{i=m_{j}}^{m_{j+1}-1}\left\|e_{u}^{i}\right\|_{0}^{2}\left(\left\|e_{u}^{i+1}\right\|_{2}^{2}+\left\|u\left(t_{i+1}\right)\right\|_{2}^{2}\right)+\frac{1}{8} \mu \Delta t \sum_{i=m_{j}}^{m_{j+1}-1}\left\|\nabla e^{i+1}\right\|_{0}^{2},
\end{aligned}
$$




$$
\begin{gathered}
R_{5}+R_{7} \leq c \Delta t \sum_{i=m_{j}}^{m_{j+1}-1}\left\|u\left(t_{i}\right)-u\left(t_{i+1}\right)\right\|_{0}^{2}\left(\left\|u\left(t_{i+1}\right)\right\|_{2}^{2}+\left\|e_{u}^{i+1}\right\|_{2}^{2}\right) \\
+\frac{1}{8} \mu \Delta t \sum_{i=m_{j}}^{m_{j+1}-1}\left\|\nabla e^{i+1}\right\|_{0}^{2}, \\
R_{6} \leq c \Delta t \sum_{i=m_{j}}^{m_{j+1}-1}\left\|u\left(t_{i+1}\right)\right\|_{2}^{2}\left\|e_{u}^{i+1}\right\|_{0}^{2}+\frac{1}{8} \mu \Delta t \sum_{i=m_{j}}^{m_{j+1}-1}\left\|\nabla e^{i+1}\right\|_{0}^{2} .
\end{gathered}
$$

Then, according to (3.2), we have

$$
\begin{aligned}
I_{1}+I_{2} \leq \frac{8 c^{*}}{\mu} \Delta t & \sum_{i=m_{j}}^{m_{j+1}-1}\left(\left\|e^{i}-e^{i+1}\right\|_{0}^{2}+\left\|e^{i+1}\right\|_{0}^{2}\right) \\
& +c \Delta t^{2} \sum_{i=m_{j}}^{m_{j+1}-1} \int_{t_{i}}^{t_{i+1}}\left\|u_{t}(t)\right\|_{0}^{2} d t \\
& +c r \Delta t h^{2(k+1)}+\frac{1}{2} \mu \Delta t \sum_{i=m_{j}}^{m_{j+1}-1}\left\|\nabla e^{i+1}\right\|_{0}^{2}
\end{aligned}
$$

where $c^{*}=\sup _{i}\left(\left\|e_{u}^{i+1}\right\|_{2}^{2}+\left\|u\left(t_{i+1}\right)\right\|_{2}^{2}\right)$. Analogously to (4.16), we obtain

$$
\begin{aligned}
& I_{6}+I_{7} \leq 2 r \Delta t\left|b\left(\frac{1}{r} \sum_{i=m_{j}}^{m_{j+1}-1} e^{i}, W\left(t_{m_{j+1}}\right), \xi^{m_{j+1}}\right)\right| \\
&+2 r \Delta t\left|b\left(\frac{1}{r} \sum_{i=m_{j}}^{m_{j+1}-1}\left(\tilde{u}^{i}-\tilde{u}^{m_{j}}\right), \xi_{W}^{m_{j+1}}, \xi^{m_{j+1}}\right)\right| \\
&+2 r \Delta t\left|b\left(\frac{1}{r} \sum_{i=m_{j}}^{m_{j+1}-1} e^{i}, \xi_{W}^{m_{j+1}}, \xi^{m_{j+1}}\right)\right| \\
&+2 r \Delta t\left|b\left(\frac{1}{r} \sum_{i=m_{j}}^{m_{j+1}-1}\left(\tilde{u}^{i}-\tilde{u}^{m_{j}}\right), W\left(t_{m_{j+1}}\right), \xi^{m_{j+1}}\right)\right| \\
&+2 r \Delta t\left|b\left(\tilde{u}^{m_{j}}-u\left(t_{m_{j}}\right), \xi_{W}^{m_{j+1}}, \xi^{m_{j+1}}\right)\right| \\
&+2 r \Delta t\left|b\left(\tilde{u}^{m_{j}}-u\left(t_{m_{j}}\right), W\left(t_{m_{j+1}}\right), \xi^{m_{j+1}}\right)\right| \\
&+ 2 r \Delta t\left|b\left(u\left(t_{m_{j}}\right), \xi_{W}^{m_{j+1}}, \xi^{m_{j+1}}\right)\right| \\
&+2 r \Delta t\left|b\left(u\left(t_{m_{j}}\right)-u\left(t_{m_{j+1}}\right), W\left(t_{m_{j+1}}\right), \xi^{m_{j+1}}\right)\right| \\
& \leq \frac{8 c^{* *}}{r \gamma} \Delta t \sum_{i=m_{j}}^{m_{j+1}-1}\left(\left\|e^{i}-e^{i+1}\right\|_{0}^{2}+\left\|e^{i+1}\right\|_{0}^{2}\right)+c \Delta t \sum_{i=m_{j}}^{m_{j+1}-1}\left\|\tilde{u}^{i}-\tilde{u}^{m_{j}}\right\|_{0}^{2} \\
&+\frac{1}{2} r \Delta t \gamma\left\|\nabla \xi^{m_{j+1}}\right\|_{0}^{2}+c(r \Delta t)^{2} \int_{t_{m_{j}}}^{t_{m_{j+1}}}\left\|u_{t}(t)\right\|_{0}^{2} d t+c r \Delta t h^{2(k+1)},
\end{aligned}
$$

where $c^{* *}=\left\|\xi_{W}^{m_{j+1}}\right\|_{2}^{2}+\left\|W\left(t_{m_{j+1}}\right)\right\|_{2}^{2}$. 
Combining (4.11), (4.14), (4.15), (4.16) and (4.17) yields

$$
\begin{aligned}
& \left\|e^{m_{j+1}}\right\|_{0}^{2}-\left\|e^{m_{j}}\right\|_{0}^{2}+\sum_{i=m_{j}}^{m_{j+1}-1}\left\|e^{i+1}-e^{i}\right\|_{0}^{2} \\
& +\Delta t(\mu+\kappa) \sum_{i=m_{j}}^{m_{j+1}-1}\left\|\nabla e^{i+1}\right\|_{0}^{2} \\
& +\left\|\xi^{m_{j+1}}\right\|_{0}^{2}-\left\|\xi^{m_{j}}\right\|_{0}^{2}+\left\|\xi^{m_{j+1}}-\xi^{m_{j}}\right\|_{0}^{2} \\
& +r \Delta t \gamma\left\|\nabla \xi^{m_{j+1}}\right\|_{0}^{2}+8 r \Delta t \kappa\left\|\xi^{m_{j+1}}\right\|_{0}^{2} \\
& \leq c \Delta t \sum_{i=m_{j}}^{m_{j+1}-1}\left\|\Pi_{u, t}^{i+1}\right\|_{0}^{2}+c r \Delta t\left\|\Pi_{W, s}^{m_{j+1}}\right\|_{0}^{2} \\
& +c \Delta t \sum_{i=m_{j}}^{m_{j+1}-1}\left(\left\|\tilde{W}^{i}-\tilde{W}^{m_{j}}\right\|_{0}^{2}+\left\|\tilde{u}^{i}-\tilde{u}^{m_{j}}\right\|_{0}^{2}\right) \\
& +c r^{2} \Delta t^{2}\left(\sum_{i=m_{j}}^{m_{j+1}-1} \int_{t_{i}}^{t_{i+1}}\left\|W_{t}(t)\right\|_{0}^{2} d t+\int_{t_{m_{j}}}^{t_{m_{j+1}-1}}\left\|u_{t}(t)\right\|_{0}^{2} d t\right) \\
& +c r \Delta t h^{2(k+1)}+4 \Delta t \kappa \sum_{i=m_{j}}^{m_{j+1}-1}\left(\left\|\xi^{m_{j}}-\xi^{m_{j+1}}\right\|_{0}^{2}+\left\|\xi^{m_{j+1}}\right\|_{0}^{2}\right) \\
& +\left(\frac{16 \kappa^{2}}{\gamma}+\frac{8 c^{* *}}{r \gamma}+\frac{8 c^{*}}{\mu}\right) \Delta t \sum_{i=m_{j}}^{m_{j+1}-1}\left(\left\|e^{i}-e^{i+1}\right\|_{0}^{2}+\left\|e^{i+1}\right\|_{0}^{2}\right) .
\end{aligned}
$$

Let $4 \Delta t \kappa \leq 1$ and $\left(\frac{16 \kappa^{2}}{\gamma}+\frac{8 c^{* *}}{r \gamma}+\frac{8 c^{*}}{\mu}\right) \Delta t \leq 1$. Then, (4.18) can be rewritten as

$$
\begin{aligned}
& \left\|e^{m_{j+1}}\right\|_{0}^{2}-\left\|e^{m_{j}}\right\|_{0}^{2} \\
& +\Delta t(\mu+\kappa) \sum_{i=m_{j}}^{m_{j+1}-1}\left\|\nabla e^{i+1}\right\|_{0}^{2}+\left\|\xi^{m_{j+1}}\right\|_{0}^{2}-\left\|\xi^{m_{j}}\right\|_{0}^{2} \\
& +r \Delta t \gamma\left\|\nabla \xi^{m_{j+1}}\right\|_{0}^{2}+8 r \Delta t \kappa\left\|\xi^{m_{j+1}}\right\|_{0}^{2} \\
& \leq c \Delta t \sum_{i=m_{j}}^{m_{j+1}-1}\left\|\Pi_{u, t}^{i+1}\right\|_{0}^{2}+c r \Delta t\left\|\Pi_{W, s}^{m_{j+1}}\right\|_{0}^{2} \\
& +c \Delta t \sum_{i=m_{j}}^{m_{j+1}-1}\left(\left\|\tilde{W}^{i}-\tilde{W}^{m_{j}}\right\|_{0}^{2}+\left\|\tilde{u}^{i}-\tilde{u}^{m_{j}}\right\|_{0}^{2}\right) \\
& +c r^{2} \Delta t^{2}\left(\sum_{i=m_{j}}^{m_{j+1}-1} \int_{t_{i}}^{t_{i+1}}\left\|W_{t}(t)\right\|_{0}^{2} d t+\int_{t_{m_{j}}}^{t_{m_{j+1}-1}}\left\|u_{t}(t)\right\|_{0}^{2} d t\right) \\
& +c r \Delta t h^{2(k+1)}+c \Delta t \sum_{i=m_{j}}^{m_{j+1}-1}\left(\left\|\xi^{m_{j+1}}\right\|_{0}^{2}+\left\|e^{i+1}\right\|_{0}^{2}\right) .
\end{aligned}
$$


Sum (4.19) over $j=0,1, \ldots, l$. Then,

$$
\begin{aligned}
& \left\|e^{m_{l+1}}\right\|_{0}^{2}+\Delta t(\mu+\kappa) \sum_{j=0}^{l} \sum_{i=m_{j}}^{m_{j+1}-1}\left\|\nabla e^{i+1}\right\|_{0}^{2}+\left\|\xi^{m_{l+1}}\right\|_{0}^{2} \\
& +r \Delta t \gamma \sum_{j=0}^{l}\left\|\nabla \xi^{m_{j+1}}\right\|_{0}^{2}+8 r \Delta t \kappa \sum_{j=0}^{l}\left\|\xi^{m_{j+1}}\right\|_{0}^{2} \\
& \leq c \Delta t \sum_{j=0}^{l} \sum_{i=m_{j}}^{m_{j+1}-1}\left\|\Pi_{u, t}^{i+1}\right\|_{0}^{2}+c r \Delta t \sum_{j=0}^{l}\left\|\Pi_{W, s}^{m_{j+1}}\right\|_{0}^{2}+c \Delta t^{2}+c h^{2(k+1)} \\
& +c \Delta t \sum_{j=0}^{l} \sum_{i=m_{j}}^{m_{j+1}-1}\left(\left\|\xi^{m_{j+1}}\right\|_{0}^{2}+\left\|e^{i+1}\right\|_{0}^{2}\right) \\
& +c \Delta t \sum_{j=0}^{l} \sum_{i=m_{j}}^{m_{j+1}-1}\left(\left\|\tilde{W}^{i}-\tilde{W}^{m_{j}}\right\|_{0}^{2}+\left\|\tilde{u}^{i}-\tilde{u}^{m_{j}}\right\|_{0}^{2}\right) .
\end{aligned}
$$

We now seek to estimate all terms on the right-hand side in the above inequality. Using (4.1)-(4.2) and (3.2), we have

$$
\begin{gathered}
c \Delta t \sum_{j=0}^{l} \sum_{i=m_{j}}^{m_{j+1}-1}\left\|\Pi_{u, t}^{i+1}\right\|_{0}^{2}+c r \Delta t \sum_{j=0}^{l}\left\|\Pi_{W, s}^{m_{j+1}}\right\|_{0}^{2} \\
\leq c \Delta t \sum_{j=0}^{l} \sum_{i=m_{j}}^{m_{j+1}-1}\left(\frac{1}{\Delta t} \int_{t_{i}}^{t_{i+1}}\left\|\left(P_{h}^{\omega}-I\right) u_{t}(t)\right\|_{0}^{2} d t\right. \\
\left.\quad+\Delta t \int_{t_{i}}^{t_{i+1}}\left\|u_{t t}(t)\right\|_{0}^{2} d t\right) \\
+c r \Delta t \sum_{j=0}^{l}\left(\frac{1}{r \Delta t} \int_{t_{m_{j}}}^{t_{m_{j+1}}}\left\|\left(P_{h}^{\omega}-I\right) W_{t}(t)\right\|_{0}^{2} d t\right. \\
\left.\quad+r \Delta t \int_{t_{m_{j}}}^{t_{m_{j+1}}}\left\|W_{t t}(t)\right\|_{0}^{2} d t\right) \\
\leq c\left(\int_{0}^{T}\left\|\left(P_{h}^{\omega}-I\right) u_{t}(t)\right\|_{0}^{2} d t+\Delta t^{2} \int_{0}^{T}\left\|u_{t t}(t)\right\|_{0}^{2} d t\right) \\
\quad+c\left(\int_{0}^{T}\left\|\left(P_{h}^{\omega}-I\right) W_{t}(t)\right\|_{0}^{2} d t+(r \Delta t)^{2} \int_{0}^{T}\left\|W_{t t}(t)\right\|_{0}^{2} d t\right) \\
\leq c \Delta t^{2}+c h^{2(k+1)} .
\end{gathered}
$$

Besides that,

$$
\begin{array}{r}
c \Delta t \sum_{j=0}^{l} \sum_{i=m_{j}}^{m_{j+1}-1}\left\|\tilde{W}^{i}-\tilde{W}^{m_{j}}\right\|_{0}^{2}+c \Delta t \sum_{j=0}^{l} \sum_{i=m_{j}}^{m_{j+1}-1}\left\|\tilde{u}^{i}-\tilde{u}^{m_{j}}\right\|_{0}^{2} \\
\quad \leq c \Delta t \sum_{j=0}^{l} \sum_{i=m_{j}}^{m_{j+1}-1}\left(\left\|\tilde{W}^{i}-\tilde{W}^{i+1}\right\|_{0}^{2}+\left\|\tilde{u}^{i}-\tilde{u}^{i+1}\right\|_{0}^{2}\right)
\end{array}
$$




$$
\leq c \Delta t^{2}\left(\int_{0}^{T}\left\|W_{t}(t)\right\|_{0}^{2} d t+\int_{0}^{T}\left\|u_{t}(t)\right\|_{0}^{2} d t\right) \leq c \Delta t^{2} .
$$

Hence, we have

$$
\begin{aligned}
& \left\|e^{m_{l+1}}\right\|_{0}^{2}+\Delta t(\mu+\kappa) \sum_{j=0}^{l} \sum_{i=m_{j}}^{m_{j+1}-1}\left\|\nabla e^{i+1}\right\|_{0}^{2} \\
& +\left\|\xi^{m_{l+1}}\right\|_{0}^{2}+r \Delta t \gamma \sum_{j=0}^{l}\left\|\nabla \xi^{m_{j+1}}\right\|_{0}^{2}+8 r \Delta t \kappa \sum_{j=0}^{l}\left\|\xi^{m_{j+1}}\right\|_{0}^{2} \\
& \leq c \Delta t^{2}+c h^{2(k+1)}+c \Delta t \sum_{j=0}^{l} \sum_{i=m_{j}}^{m_{j+1}-1}\left(\left\|\xi^{m_{j+1}}\right\|_{0}^{2}+\left\|e^{i+1}\right\|_{0}^{2}\right) .
\end{aligned}
$$

This yields the final result by using the Grönwall inequality:

$$
\begin{gathered}
\left\|e^{m_{l+1}}\right\|_{0}^{2}+\Delta t(\mu+\kappa) \sum_{j=0}^{l} \sum_{i=m_{j}}^{m_{j+1}-1}\left\|\nabla e^{i+1}\right\|_{0}^{2}+\left\|\xi^{m_{l+1}}\right\|_{0}^{2} \\
+r \Delta t \gamma \sum_{j=0}^{l}\left\|\nabla \xi^{m_{j+1}}\right\|_{0}^{2}+8 r \Delta t \kappa \sum_{j=0}^{l}\left\|\xi^{m_{j+1}}\right\|_{0}^{2} \\
\leq c \Delta t^{2}+c h^{2(k+1)} \cdot \quad \square
\end{gathered}
$$

In case of smaller time steps for the linear velocity, we have the following error estimate:

THEOREM 4.2. Under the assumption of Theorem 4.1, the following estimate holds: for $J=1,2, \ldots, r-1$ and $j=0,1, \ldots, l$,

$$
\begin{gathered}
\left\|e^{m_{j}+J+1}\right\|_{0}^{2}-\left\|e^{m_{j}}\right\|_{0}^{2}+\sum_{i=m_{j}}^{m_{j}+J}\left\|e^{i+1}-e^{i}\right\|_{0}^{2}+\Delta t(\mu+\kappa) \sum_{i=m_{j}}^{m_{j}+J}\left\|\nabla e^{i+1}\right\|_{0}^{2} \\
\leq c \Delta t^{2}+c h^{2(k+1)} .
\end{gathered}
$$

Proof. Let $\left(v_{h}, q_{h}\right)=2 \Delta t\left(e^{m+1}, \epsilon^{m+1}\right)$ in (4.6). Then, summing the resulting equation over $m=m_{j}, m_{j}+1, \ldots, m_{j}+J$ leads to

$$
\begin{gathered}
\left\|e^{m_{j}+J+1}\right\|_{0}^{2}-\left\|e^{m_{j}}\right\|_{0}^{2}+\sum_{i=m_{j}}^{m_{j}+J}\left\|e^{i+1}-e^{i}\right\|_{0}^{2}+2 \Delta t(\mu+\kappa) \sum_{i=m_{j}}^{m_{j}+J}\left\|\nabla e^{i+1}\right\|_{0}^{2} \\
=2 \Delta t \sum_{i=m_{j}}^{m_{j}+J} b\left(u_{h}^{i}, u_{h}^{i+1}, e^{i+1}\right)-2 \Delta t \sum_{i=m_{j}}^{m_{j}+J} b\left(u\left(t_{i+1}\right), u\left(t_{i+1}\right), e^{i+1}\right) \\
+2 \Delta t \kappa \sum_{i=m_{j}}^{m_{j}+J}\left(\nabla \times W\left(t_{i+1}\right), e^{i+1}\right)-4 \Delta t \kappa \sum_{i=m_{j}}^{m_{j}+J}\left(\nabla \times W_{h}^{m_{j}}, e^{i+1}\right) \\
+2 \Delta t \sum_{i=m_{j}}^{m_{j}+J}\left(\Pi_{u, t}^{i+1}, e^{i+1}\right) .
\end{gathered}
$$


Similar to (4.11), (4.14), (4.16), (4.20), and (4.21)-(4.23), we have

$$
\begin{gathered}
\left\|e^{m_{j}+J+1}\right\|_{0}^{2}-\left\|e^{m_{j}}\right\|_{0}^{2}+\sum_{i=m_{j}}^{m_{j}+J}\left\|e^{i+1}-e^{i}\right\|_{0}^{2}+\Delta t(\mu+\kappa) \sum_{i=m_{j}}^{m_{j}+J}\left\|\nabla e^{i+1}\right\|_{0}^{2} \\
\leq c \Delta t^{2}+c \Delta t h^{2(k+1)}+c r \Delta t \sum_{i=m_{j}}^{m_{j}+J}\left\|e^{i}\right\|_{0}^{2}+c r \Delta t\left\|\xi^{m_{j}}\right\|_{0}^{2} \\
+c \Delta t \sum_{i=m_{j}}^{m_{j}+J}\left\|\tilde{W}^{i}-\tilde{W}^{m_{j}}\right\|_{0}^{2}+c \Delta t \sum_{i=m_{j}}^{m_{j}+J}\left\|\Pi_{u, t}^{i+1}\right\|_{0}^{2} \\
\leq c \Delta t^{2}+c \Delta t h^{2(k+1)}+c r \Delta t \sum_{i=m_{j}}^{m_{j}+J}\left\|e^{i}\right\|_{0}^{2}+c r \Delta t\left\|\xi^{m_{j}}\right\|_{0}^{2}+c\left(\Delta t^{2}+h^{2(k+1)}\right) .
\end{gathered}
$$

Hence, we arrive at

$$
\begin{gathered}
\left\|e^{m_{j}+J+1}\right\|_{0}^{2}-\left\|e^{m_{j}}\right\|_{0}^{2}+\sum_{i=m_{j}}^{m_{j}+J}\left\|e^{i+1}-e^{i}\right\|_{0}^{2}+\Delta t(\mu+\kappa) \sum_{i=m_{j}}^{m_{j}+J}\left\|\nabla e^{i+1}\right\|_{0}^{2} \\
\leq c \Delta t^{2}+c h^{2(k+1)}+c \Delta t \sum_{i=m_{j}}^{m_{j}+J}\left\|e^{i}\right\|_{0}^{2}+c r \Delta t\left\|\xi^{m_{j}}\right\|_{0}^{2} .
\end{gathered}
$$

Then by Theorem 4.1, we have

$$
\begin{gathered}
\left\|e^{m_{j}+J+1}\right\|_{0}^{2}-\left\|e^{m_{j}}\right\|_{0}^{2}+\sum_{i=m_{j}}^{m_{j}+J}\left\|e^{i+1}-e^{i}\right\|_{0}^{2}+\Delta t(\mu+\kappa) \sum_{i=m_{j}}^{m_{j}+J}\left\|\nabla e^{i+1}\right\|_{0}^{2} \\
\leq c \Delta t^{2}+c h^{2(k+1)} \cdot
\end{gathered}
$$

Finally, using the triangle inequality, combining the approximation properties (3.2) and the above theorems, one obtains the next theorem.

THEOREM 4.3. Under the assumption of Theorem 4.1, for $j=0,1, \ldots, \bar{M}-1$ and $m=1,2, \ldots, N$, the following estimates hold:

$$
\begin{aligned}
\left\|u\left(t_{m}\right)-u_{h}^{m}\right\|_{0}^{2} & \leq c \Delta t^{2}+c h^{2(k+1)}, \\
\left\|\nabla\left(u\left(t_{m}\right)-u_{h}^{m}\right)\right\|_{0}^{2} & \leq c \Delta t^{2}+c h^{2 k}, \\
\left\|W\left(t_{m_{j+1}}\right)-W_{h}^{m_{j+1}}\right\|_{0}^{2} & \leq c \Delta t^{2}+c h^{2(k+1)}, \\
\left\|\nabla\left(W\left(t_{m_{j+1}}\right)-W_{h}^{m_{j+1}}\right)\right\|_{0}^{2} & \leq c \Delta t^{2}+c h^{2 k} .
\end{aligned}
$$

5. Computational experiments. In this section, we give some numerical experiments to verify the estimates developed in the previous sections and illustrate the efficiency of the decoupling finite element method with different time steps. On the one hand, we test a problem with known analytical solution to verify the convergence rates prediction of the proposed algorithm. On the other hand, we simulate a realistic example of the stirring of a passive scalar. In all experiments, a $\left(P_{2}, P_{1}, P_{2}\right)$-finite element pair is used to approximate the linear velocity, the pressure, and the angular velocity, respectively. 


\section{ETNA}

Kent State University and

Johann Radon Institute (RICAM)

5.1. A problem with analytical solution. In this section, we present some numerical results to show the convergence performance for the micropolar fluid model (2.1)-(2.2) for the following analytical solution:

$$
\begin{aligned}
u_{1} & =\pi \sin (t) \sin ^{2}(\pi x) \sin (2 \pi y), \quad u_{2}=\pi \sin (t) \sin (2 \pi x) \sin ^{2}(\pi y), \\
p & =\sin (t) \cos (\pi x) \sin (\pi y), \\
W_{3} & =\pi \sin (t) \sin ^{2}(\pi x) \sin ^{2}(\pi y) .
\end{aligned}
$$

The source terms $f$ and $g$ can be obtained by evaluating the equations of problem (2.1) for the analytical solution. Denote $\operatorname{Err}(s)=\left\|s\left(t_{m}\right)-s_{h}^{m}\right\|_{0}$ and $s=u, W$.

We set $\Omega=(0,1)^{2}$ and choose the parameters $\mu=\kappa=\gamma=1.0$. Moreover, we present numerical results for the errors, the convergence rates, and the CPU-times for different values of the mesh size $h$. In Table 5.1 and Table 5.2, we list the computational results with $\Delta t=h^{3}$ at the final time $T=1.0$ for $r=1$ and $r=5$, respectively. From these tables, we can find that the convergence rates are $\mathcal{O}\left(h^{3}\right)$ for the $L^{2}$-norm of $u, \mathcal{O}\left(h^{2}\right)$ for the $H^{1}$-seminorm of $u$, $\mathcal{O}\left(h^{3}\right)$ for the $L^{2}$-norm of $W$, and $\mathcal{O}\left(h^{2}\right)$ for the $H^{1}$-seminorm of $W$. This shows that the decoupled method works well, and the numerical convergence rates agree with the theoretical analysis. As expected, Algorithm 1 with $r=5$ requires less CPU-time than Algorithm 1 with $r=1$ to achieve nearly the same relative error.

TABLE 5.1

Convergence performance and CPU-time of Algorithm 1 with $r=1$.

\begin{tabular}{lccccccccr}
$1 / h$ & $\operatorname{Err}(u)$ & rate & $\operatorname{Err}(\nabla u)$ & rate & $\operatorname{Err}(W)$ & rate & $\operatorname{Err}(\nabla W)$ & rate & $C P U$ \\
\hline 5 & 0.02043 & - & 0.10769 & - & 0.01473 & - & 0.09398 & - & 2.905 \\
10 & 0.00251 & 3.02 & 0.02868 & 1.91 & 0.00171 & 3.11 & 0.02490 & 1.92 & 86.435 \\
15 & 0.00074 & 3.00 & 0.01292 & 1.97 & 0.00049 & 3.07 & 0.01121 & 1.97 & 585.296 \\
20 & 0.00031 & 3.00 & 0.00731 & 1.98 & 0.00021 & 3.04 & 0.00633 & 1.98 & 2254.59 \\
25 & 0.00016 & 3.01 & 0.00469 & 1.99 & 0.00010 & 3.03 & 0.00406 & 1.99 & 6932.17
\end{tabular}

TABLE 5.2

Convergence performance and CPU-time of Algorithm 1 with $r=5$.

\begin{tabular}{lccccccccr}
$1 / h$ & $\operatorname{Err}(u)$ & rate & $\operatorname{Err}(\nabla u)$ & rate & $\operatorname{Err}(W)$ & rate & $\operatorname{Err}(\nabla W)$ & rate & $C P U$ \\
\hline 5 & 0.02095 & - & 0.10771 & - & 0.01711 & - & 0.09426 & - & 2.268 \\
10 & 0.00255 & 3.04 & 0.02868 & 1.91 & 0.00192 & 3.16 & 0.02491 & 1.92 & 73.797 \\
15 & 0.00075 & 3.01 & 0.01293 & 1.97 & 0.00055 & 3.10 & 0.01121 & 1.97 & 512.904 \\
20 & 0.00032 & 3.01 & 0.00731 & 1.98 & 0.00023 & 3.06 & 0.00633 & 1.98 & 1891.03 \\
25 & 0.00016 & 3.01 & 0.00469 & 1.99 & 0.00011 & 3.05 & 0.00406 & 1.99 & 6013.49
\end{tabular}

5.2. Stirring of a passive scalar. In this example, we consider an important practical problem, the stirring of a passive scalar, which has been tested in [33]. In order to model the passive scalar, we combine the micropolar fluid model (2.1)-(2.2) with the following convection equation:

$$
\varphi_{t}+u \cdot \nabla \varphi=0,
$$


together with the initial condition

$$
\left.\varphi\right|_{t=0}= \begin{cases}1, & y<0, \\ 0, & y \geq 0,\end{cases}
$$

where $\varphi$ represents a passive scalar, and the linear velocity $u$ is obtained by solving the micropolar fluid model (2.1)-(2.2) using Algorithm 1.

Firstly, consider $\Omega=(-1,1)^{2}$. Set $\mu=\kappa=0.1, \gamma=2.0$, and $f=0, g=25(x-1)$. Then, the micropolar fluid model (2.1)-(2.2) is solved by Algorithm 1. Secondly, based on the known linear velocity $u$, we solve the convection equation (5.1) to obtain the passive scalar $\varphi$, where we apply the backward Euler scheme for the temporal discretization and $P_{2}$-finite elements for the spatial discretization with a time step $\Delta t=0.01$ and a mesh size $h=\frac{1}{48}$.

In Figures 5.1 and 5.2, we plot the evolution of the passive scalar with $r=1$ and $r=5$, respectively. From these figures, one can find that $\varphi$ has almost the same trend when Algorithm 1 is applied with $r=1$ and $r=5$. However, Algorithm 1 with $r=5$ can save much computational time. Moreover, it is found that the results of our algorithm are similar to those in $[15,33,42]$. Therefore, Algorithm 1 captures this model well.
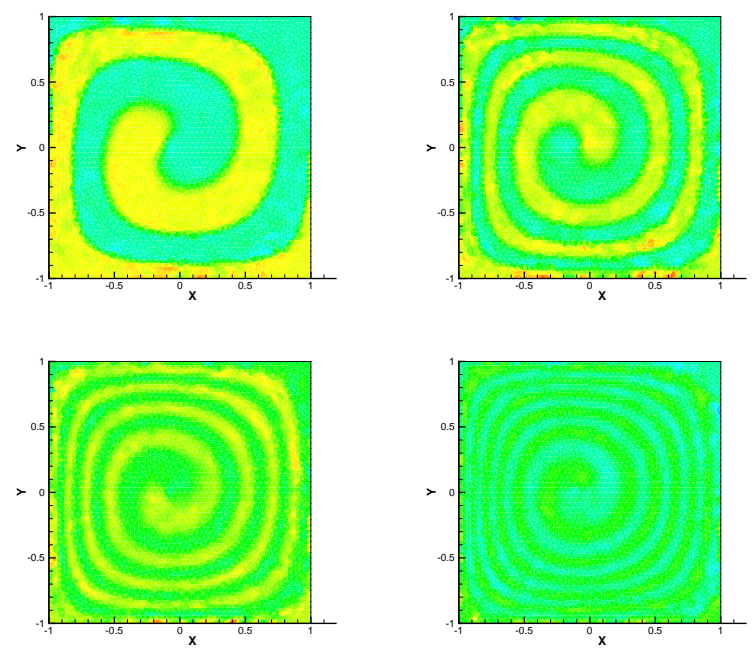

FIG. 5.1. The evolution of the passive scalar $\varphi$ for $T=10,20,30,40$ when $r=1$.

6. Conclusions. In this work we have presented a decoupling finite element method with different time steps for solving the micropolar fluid model. The main feature of our method is a decoupling of the linear velocity and the angular velocity at each time step and the use of a small time step for the linear velocity $u$ and the pressure $p$ and a large time step for the angular velocity $W$. This has more efficiency compared with a decoupled algorithm with equal time steps. Numerical tests are presented to confirm that the decoupling finite element method with different time steps is efficient.

The future extension of this work lies in the solutions of the magneto-micropolar fluid equations (Maxwell equations coupled with (2.1)), the equations of ferrohydrodynamics (magnetostatic equations coupled with (2.1)), and the flow and heat transfer of micropolar fluid equations (heat equations coupled with (2.1)). The design, analysis, and implementation 


\section{ETNA}

Kent State University and

Johann Radon Institute (RICAM)
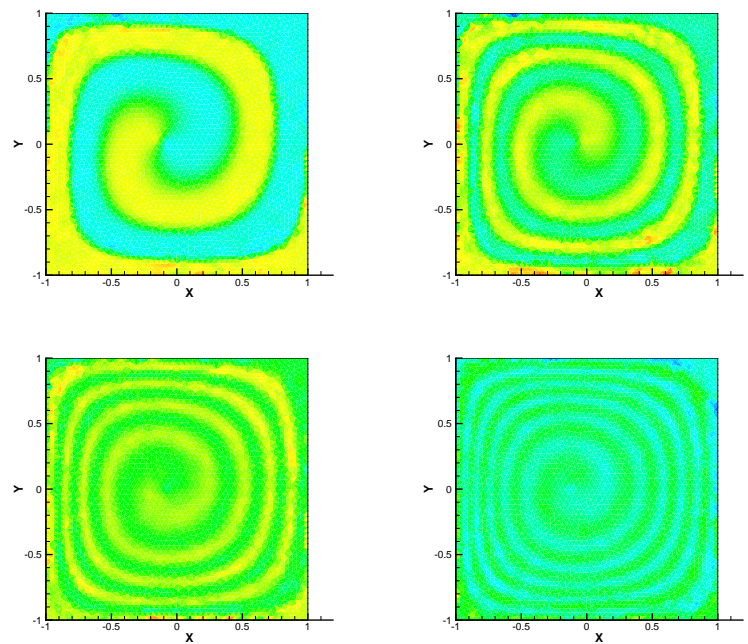

FIG. 5.2. The evolution of the passive scalar $\varphi$ for $T=10,20,30,40$ when $r=5$.

of the decoupling finite element algorithm with different time steps for these problems may be more complicated than for those presented here, but they will allow for more interesting and realistic simulations.

Acknowledgement. The authors would like to thank the editor and the anonymous referees for their helpful comments and suggestions which helped to improve the quality of the present paper. This work is supported by the Natural Science Foundation of Xinjiang Province (grant number 2021D01E11).

\section{REFERENCES}

[1] R. S. Agarwal And C. Dhanapal, Numerical solutions to the flow of a micropolar fluid between porous walls of different permeability, Internat. J. Engrg. Sci., 25 (1987), pp. 325-336.

[2] M. Ashraf, M. A. KAMAL, AND K. S. SYED, Numerical study of asymmetric laminar flow of micropolar fluids in a porous channel, Comput. \& Fluids, 38 (2009), pp. 1895-1902.

[3] J. L. Boldrini, E. Notte-Cuello, M. Poblete-Cantellano, L. Friz, and M. A. Rojas-Medar, Pointwise error estimate for spectral Galerkin approximations of micropolar equations, Numer. Funct. Anal. Optim., 37 (2016), pp. 304-323.

[4] J. L. BOLDRINI AND M. A. ROJAS-MEDAR, On the convergence rate of spectral approximation for the equations for nonhomogeneous asymmetric fluids, RAIRO Modél. Math. Anal. Numér., 30 (1996), pp. 123-155.

[5] J. L. Boldrini, M. A. Rojas-Medar, ANd E. FernándeZ-CARA, Semi-Galerkin approximation and strong solutions to the equations of the nonhomogeneous asymmetric fluids, J. Math. Pures Appl., 82 (2003), pp. 1499-1525.

[6] Z. CHEN AND W. PRICE, Decay estimates of linearized micropolar fluid flows in $R^{3}$ space with applications to $L^{3}$-strong solutions, Internat. J. Engrg. Sci., 44 (2006), pp. 859-873.

[7] J. M. CONNORS AND J. S. Howell, A fluid-fluid interaction method using decoupled subproblems and differing time steps, Numer. Methods Partial Differential Equations, 28 (2012), pp. 1283-1308.

[8] C. DAWSON AND R. KIRBY, High resolution schemes for conservation laws with locally varying time steps, SIAM J. Sci. Comput., 22 (2001), pp. 2256-2281.

[9] B. DONG AND Z. ZHANG, Global regularity of the 2D micropolar fluid flows with zero angular viscosity, J. Differential Equations, 249 (2010), pp. 200-213.

[10] A. C. ERIngEn, Simple microfluids, Internat. J. Engrg. Sci., 2 (1964), pp. 205-217.

[11] - Theory of micropolar fluids, J. Math. Mech., 16 (1966), pp. 1-18. 
[12] G. P. GALDI AND S. RiONERO, A note on the existence and uniqueness of solutions of the micropolar fluid equations, Internat. J. Engrg. Sci., 15 (1977), pp. 105-108.

[13] Z. GE AND M. MA, Multirate iterative scheme based on multiphysics discontinuous Galerkin method for a poroelasticity model, Appl. Numer. Math., 128 (2018), pp. 125-138.

[14] H. JIA, P. SHI, AND K. LI, A decoupling method with different subdomain time steps for the non-stationary Navier-Stokes/Darcy model, J. Comput. Math., 35 (2017), pp. 319-345.

[15] Y. JIANG AND Y. YANG, Analysis of some projection methods for the incompressible fluids with microstructure, J. Korean Math. Soc., 55 (2018), pp. 471-506.

[16] M. A. KAMAL, M. ASHRAF, AND K. S. SYED, Numerical solution of steady viscous flow of a micropolar fluid driven by injection between two porous disks, Appl. Math. Comput., 179 (2006), pp. 1-10.

[17] R. KIRBY, On the convergence of high resolution methods with multiple time scales for hyperbolic conservation laws, Math. Comp., 72 (2002), pp. 1239-1250.

[18] H. LANGE, The existence of instationary flows in incompressible micropolar fluids, Arch. Mech., 29 (1977), pp. 741-744.

[19] R. LI, J. CHEN, Z. CHEN, AND Y. GAO, A decoupled scheme with leap-frog multi-time step for non-stationary Stokes-Darcy system, Int. J. Comput. Math., 95 (2018), pp. 361-381.

[20] Y. LI AND Y. HoU, A second-order partitioned method with different subdomain time steps for the evolutionary Stokes-Darcy system, Math. Methods Appl. Sci., 41 (2018), pp. 2178-2208.

[21] T. LI, P. HuAnG, AND Y. HE, A fully discrete, decoupled scheme with different time steps for approximating nematic liquid crystal flow, Int. J. Numer. Anal. Model., 18 (2021), pp. 811-833.

[22] C. Liao, P. HuAng, AND Y. HE, A decoupled finite element method with different time steps for the nonstationary Darcy-Brinkman problem, J. Numer. Math., 28 (2020), pp. 33-62.

[23] G. ŁUKASZEWICZ, On nonstationary flows of incompressible asymmetric fluids, Math. Methods Appl. Sci., 13 (1990), pp. 219-232.

[24] - Micropolar Fluids: Theory and Applications, Birkhäuser, Boston, 1999.

[25] _ L Long time behavior of 2D micropolar fluid flows, Math. Comput. Modelling, 34 (2001), pp. 487-509.

[26] R. H. NochetTo, A. J. SALGADO, AND I. TOMAS, The micropolar Navier-Stokes equations: a priori error analysis, Math. Models Methods Appl. Sci.. 24 (2014), pp. 1237-1264.

[27] E. ORTEGA-TORRES AND M. ROJAS-MEDAR, Optimal error estimate of the penalty finite element method for the micropolar fluid equations, Numer. Funct. Anal. Optim., 29 (2008), pp. 612-637.

[28] S. OSHER AND R. SANDERS, Numerical approximations to nonlinear conservation laws with locally varying time and space grids, Math. Comp., 41 (1983), pp. 321-336.

[29] I. PAPAutsky, J. BRAZZLE, T. AMEel, AND A. B. FraZIER, Laminar fluid behavior in microchannels using micropolar fluid theory, Sens. Actuators A Phys., 73 (1999), pp. 101-108.

[30] S. K. L. RAO, Stability of micropolar fluid motions, Internat. J. Engrg. Sci., 8 (1970), pp. 753-762.

[31] S. S. RAVINDRAN, Analysis of a decoupled time-stepping scheme for evolutionary micropolar fluid flows, Adv. Numer. Anal., 2016, Art. 7010645, 13 pages.

[32] M. A. Rojas-Medar, Magneto-micropolar fuid motion: existence and uniqueness of strong solution, Math. Nachr., 188 (1997), pp. 301-319.

[33] A. J. SALGADO, Convergence analysis of fractional time-stepping techniques for incompressible luids with microstructure, J. Sci. Comput., 64 (2015), pp. 216-233.

[34] V. A. SAVA, The initial-boundary-value problems in the theory of micropolar fluids, Z. Angew. Math. Mech., 58 (1978), pp. 511-518.

[35] V. SAVCENCO, W. HUNDSDORFER, AND J. G. VERWER, A multirate time stepping strategy for stiff ordinary differential equations, BIT, 47 (2007), pp. 137-155.

[36] L. Shan, H. ZHENG, AND W. J. LAYTON, A decoupling method with different subdomain time steps for the nonstationary Stokes-Darcy model, Numer. Methods Partial Differential Equations, 29 (2013), pp. 549-583.

[37] I. Shevchenko, M. Kaltenbacher, AND B. Wohlmuth, A multi-time stepping integration method for the ultrasound heating problem, Z. Angew. Math. Mech., 92 (2012), pp. 869-881.

[38] F. SHI, G. LIANG, Y. ZHAO, AND J. ZOU, New splitting methods for time-dependent convection-dominated diffusion problems, Commun. Comput. Phys., 16 (2014), pp. 1239-1262.

[39] F. SHI, H. ZHENG, Y. CAO, J. LI, AND R. ZhaO, A fast numerical method for solving coupled Burgers' equations, Numer. Methods Partial Differential Equations, 33 (2017), pp. 1823-1838.

[40] P. Wilding, J. Pfahler, H. H. BaU, J. N. Zemel, AND L. J. KRICKa, Manipulation and flow of biological fluids in straight channels micromachined in silicon, Clin. Chem., 40 (1994), pp. 43-47.

[41] D. XUE AND Y. HoU, A second-order decoupled algorithm with different subdomain time steps for the non-stationary Stokes/Darcy model, Numer. Algorithms, 88 (2021), pp. 1137-1182.

[42] Y. YANG AND Y. JIANG, Analysis of two decoupled time-stepping finite element methods for incompressible fluids with microstructure, Int. J. Comput. Math., 95 (2018), pp. 686-709.

[43] B. YuAn, On regularity criteria for weak solutions to the micropolar fluid equations in Lorentz space, Proc. Amer. Math. Soc., 138 (2010), pp. 2025-2036. 